\title{
Density estimation with minimization of $U$-divergence
}

\author{
Kanta Naito • Shinto Eguchi
}

Received: 10 June 2011 / Accepted: 9 May 2012 / Published online: 31 May 2012

(C) The Author(s) 2012

\begin{abstract}
This paper is concerned with density estimation based on the stagewise minimization of the $U$-divergence. The $U$-divergence is a general divergence measure involving a convex function $U$ which includes the Kullback-Leibler divergence and the $L_{2}$ norm as special cases. The algorithm to yield the density estimator is closely related to the boosting algorithm and it is shown that the usual kernel density estimator can also be seen as a special case of the proposed estimator. Non-asymptotic error bounds of the proposed estimators are developed and numerical experiments show that the proposed estimators often perform better than several existing methods for density estimation.
\end{abstract}

Keywords Density estimation · Boosting $\cdot$ Stagewise minimization $\cdot U$-divergence

\section{Introduction}

Recently, there has been renewed widespread interest in supervised learning in regard to regression, classification and pattern recognition. Boosting has been known as a promising technique with feasible computational algorithms that have received a great deal of attention. Boosting attempts to create a strong committee from a suitable combination of weak learners, and was first proposed by Schapire (1990). Subsequent studies on boosting are summarized in Bishop (2006) and Hastie et al. (2009).

In contrast to supervised learning, boosting approaches to unsupervised learning, such as density estimation, appear to be less developed. Although it is understood that unsupervised learning is more difficult than supervised learning, there is a need for an effective learning method for density estimation. The purpose of this study is to develop a general

Editor: James Cussens.

K. Naito $(\bowtie)$

Department of Mathematics, Shimane University, Matsue 690-8504, Japan

e-mail: naito@riko.shimane-u.ac.jp

S. Eguchi

The Institute of Statistical Mathematics, 10-3 Midori-cho, Tachikawa, Tokyo 190-8562, Japan 
but practical learning method for multivariate density estimation. There have been several reports on stagewise optimization algorithms like boosting for density estimation, including Friedman et al. (1984), Ridgeway (2002), Rosset and Segal (2002) and Klemelä (2007). The approaches used in these studies are based on implementing repeat optimization of a certain loss function which can be seen as an empirical form of the corresponding divergence measure: the Kullback-Leibler divergence and the $L_{2}$ norm. A simple and effective stagewise optimization method based on the $L_{2}$ norm has been fully discussed in Klemelä (2007), in which non-asymptotic error bounds for the estimator were developed. The method is to find a fine linear combination of words in a dictionary via the stagewise optimization of the empirical $L_{2}$ norm.

Let $f(x)$ be a probability density function on $\mathbb{R}^{d}$. Assume that random vectors $X_{1}, \ldots, X_{n}$ are independently and identically distributed with $f(x)$. Our goal is to estimate $f(x)$ based on $X_{1}, \ldots, X_{n}$. In this paper, we consider a class of loss functions defined by convex function, called the $U$-loss function. The $U$-loss function can be obtained as an empirical form of a divergence, called the $U$-divergence. Geometry on a statistical manifold has been discussed in depth by many authors, and divergence is known to play an important role. The $U$-divergence is associated with a convex function $U$ satisfying some conditions and is known to be a useful tool for developing a variety of geometries in statistical problems. Application of the $U$-loss function to pattern recognition is described in Murata et al. (2004) and references therein. Since the usual $L_{2}$ norm can be considered to be a special case of $U$, results for unsupervised learning based on the $L_{2}$ norm can be generalized into those based on the $U$-divergence. The present research attempts to generalize the work by Klemelä (2007) in density estimation. Specifically, a density estimator obtained through the stagewise minimization of the $U$-loss function is proposed and its non-asymptotic error bounds are developed. This generalization is not straightforward and requires a considerable amount of mathematical manipulation. The use of $U$-divergence has the potential to extend theories based on the $L_{2}$ norm in statistical learning.

This paper is organized as follows. In Sect. 2 the concept of $U$-divergence is introduced, where it will be shown that some common divergences are special cases of $U$-divergence. The induced $U$-loss function, which is optimized to develop the proposed density estimator, is also addressed. Section 3 describes the algorithm by which the proposed estimator is obtained. An interesting interpretation of the algorithm is then considered from the view point of regularization. The connection between the present estimator and those that have been discussed so far, such as the kernel density estimator and boosting estimator, is discussed. The error bounds of the present estimator are developed in Sect. 4, and those for the normalized form of the estimator are also derived. All results are for so-called nonasymptotic error bounds, and some constants involved in the bounds are clarified for typical divergences. Section 5 reports on the numerical properties of the estimators. A simulation for the estimation of bivariate normal mixture densities is carried out and the results reveal that even with a simple setup the proposed estimator is superior to or at least competitive with several existing methods for density estimation. Discussions and future perspectives are presented in Sect. 6. The Appendix provides proofs of the theorems used in Sect. 4 and of technical lemmas.

\section{$2 U$-divergence and $U$-loss function}

The aim of this section is to address $U$-divergence, which plays an important role in our theory. A more detailed discussion of $U$-divergence in conjunction with the effective use of divergence in information geometry and machine learning can be found in Eguchi (2008). 


\section{$2.1 U$-divergence}

Let $\mathcal{M}$ be a space of all the nonnegative functions on a data space $\mathbb{R}^{d}$. We construct indices of information divergence on the space $\mathcal{M}$ employing properties of conjugate convexity. Let $U$ be a convex and strictly increasing function on the nonnegative real line with the derivative function $u=U^{\prime}$.

Then the conjugate convex function $\Xi$ of $U$ (Rockafeller 1996) is given by

$$
\Xi(s)=\max _{t \in \mathbb{R}}\{s t-U(t)\}
$$

which is written as $\Xi(s)=s \xi(s)-U(\xi(s))$, where $\xi(s)$ is the inverse of the function $u(t)$. Thus, $\Xi(s)$ is a primitive function of $\xi$. In what follows, we always associate $U$ with a derived triplet $(U, u, \xi)$.

The $U$-divergence on $\mathcal{M}$ between densities $f$ and $g$ is defined by

$$
\begin{aligned}
D_{U}(f, g) & =\int_{\mathbb{R}^{d}}[\Xi(f(x))-\{f(x) \xi(g(x))-U(\xi(g(x)))\}] d x \\
& =\int_{\mathbb{R}^{d}}[U(\xi(g(x)))-U(\xi(f(x)))-f(x)\{\xi(g(x))-\xi(f(x))\}] d x .
\end{aligned}
$$

Based on the properties of the convex function, $D_{U}(f, g) \geq 0$ and the equality holds if and only if $f=g$ except the set of measure zero. Typical examples of $U$-divergence are given below.

Example 1 (The Kullback-Leibler divergence) One of the most typical examples is $U(t)=$ $\exp (t)$ with the derivative $u(t)=\exp (t)$ and its inverse $\xi(u)=\log (u)$. Thus, the $U$ divergence corresponding to this choice is

$$
\begin{aligned}
D_{U}(f, g) & =\int_{\mathbb{R}^{d}}[g(x)-f(x)-f(x)\{\log g(x)-\log f(x)\}] d x \\
& =\int_{\mathbb{R}^{d}} f(x) \log \left(\frac{f(x)}{g(x)}\right) d x,
\end{aligned}
$$

which is nothing but the Kullback-Leibler divergence.

Example 2 ( $\beta$-power divergence) A second typical example is

$$
U_{\beta}(t)=\frac{1}{\beta+1}(1+\beta t)^{\frac{\beta+1}{\beta}}
$$

for a tuning parameter $0<\beta \leq 1$ and the derivative and its inverse are thus given as

$$
u_{\beta}(t)=(1+\beta t)^{\frac{1}{\beta}} \quad \text { and } \quad \xi_{\beta}(u)=\frac{u^{\beta}-1}{\beta},
$$

respectively. Noting that $U_{\beta}\left(\xi_{\beta}(u)\right)=u^{\beta+1} /(\beta+1)$, we get the resulting divergence

$$
D_{\beta}(f, g)=\frac{1}{\beta+1} \int_{\mathbb{R}^{d}}\left\{g(x)^{\beta+1}-f(x)^{\beta+1}\right\} d x-\frac{1}{\beta} \int_{\mathbb{R}^{d}} f(x)\left\{g(x)^{\beta}-f(x)^{\beta}\right\} d x,
$$


which is called the $\beta$-power divergence (Basu et al. 1998; Minami and Eguchi 2002). We notice that $\lim _{\beta \downarrow 0} U_{\beta}(t)=\exp (t)$, which implies the limit of the $\beta$-divergence reduces to the Kullback-Leibler divergence as $\beta$ goes to 0 . Alternatively, when $\beta=1, D_{\beta}(f, g)=\frac{1}{2} \| f-$ $g \|^{2}$, where \|\| denotes the $L_{2}$-norm on $\mathcal{M}$, see Scott (2001) for the statistical argument.

\section{$2.2 U$-loss function}

We consider a loss function derived naturally from the $U$-divergence. The empirical $U$-loss function for a density $g$ is defined by

$$
\widehat{L}_{U}(g)=-\frac{1}{n} \sum_{i=1}^{n} \xi\left(g\left(X_{i}\right)\right)+\int_{\mathbb{R}^{d}} U(\xi(g(x))) d x .
$$

Obviously, this is the empirical form of

$$
L_{U}(g)=-\int_{\mathbb{R}^{d}} \xi(g(x)) f(x) d x+\int_{\mathbb{R}^{d}} U(\xi(g(x))) d x,
$$

which is a part of $D_{U}(f, g)$ relating to $g$. Hence, minimization of $L_{U}(g)$ with respect to $g$ is equivalent to that of $D_{U}(f, g)$ for a fixed $f$. We aim to obtain an estimator of $f$ through the minimization of $\widehat{L}_{U}$ in the following discussion.

\subsection{Advantage of $U$-divergence}

A class of $U$-divergence directly yields a variety of density estimators. Specifically the $L_{2}$ distance produces a density estimator via additive forward learning, on the other hand density estimators can be constructed by multiplicative learning if the Kullback-Leibler distance is adopted. We could connect the $L_{2}$ distance $(\beta=1)$ and the Kullback-Leibler distance $(\beta \rightarrow 0)$ smoothly by one parameter $\beta$, if the $\beta$-power divergence is adopted. By which, we can realize various methods of learning, involving those two typical and representative learning methods.

As an another possible divergence, we might utilize $f$-divergence (see, Devroye et al. 1996) for density estimation problem. It is, however, not easy to make the empirical loss function from $f$-divergence except in the case of the Kullback-Leibler divergence. On the other hand, it is easy to express the empirical loss function from $U$-divergence, since it is a functional of the empirical distribution function. In this regard, we prefer to utilize $U$ divergence rather than $f$-divergence.

\section{Estimator and algorithm}

This section describes the method proposed to obtain the estimator and its practical algorithm.

\subsection{Form of the estimator}

Let $\mathcal{D}$ be a dictionary: a collection of densities (words). Any special requirements are not imposed on densities in $\mathcal{D}$, but we only assume smooth and bounded densities on $\mathbb{R}^{d}$ as elements of $\mathcal{D}$. And further, in this paper, we utilize a dictionary with finite cardinality. 
Let $\xi(\mathcal{D})=\{\xi(\phi) \mid \phi \in \mathcal{D}\}$ be the transformed dictionary. We define the convex hull of $\xi(\mathcal{D})$ as

$$
\operatorname{co}(\xi(\mathcal{D}))=\left\{\sum_{i=1}^{k} \lambda_{i} \xi\left(\phi_{i}\right) \mid \phi_{i} \in \mathcal{D}, \sum_{i=1}^{k} \lambda_{i}=1, \lambda_{i} \geq 0, \text { and } k=1,2, \ldots\right\} .
$$

The proposed estimator is derived by using the triplet $(U, u, \xi)$, and its general form is

$$
u\left(\sum_{t=1}^{K} \theta_{t} \xi\left(\phi_{t}(x)\right)\right), \quad x \in \mathbb{R}^{d},
$$

where $\theta_{t} \geq 0, t=1, \ldots, K, \sum_{t=1}^{K} \theta_{t}=1$ and $\phi_{t} \in \mathcal{D}, t=1, \ldots, K$. Thus, a convex combination of densities transformed by $\xi$ is first produced, and the inverse of this is then produced using $u=\xi^{-1}$.

\subsection{Algorithm}

The algorithm used to obtain the estimator is now described. Let $\varepsilon>0$ be the approximation bound. Using $u=\xi^{-1}$ and the mixing coefficients $0<\pi_{k}<1(k=1, \ldots, M-1)$, the algorithm for the stagewise minimization estimator consists of the following steps:

Step 1 Choose $\tilde{f}_{0} \in \mathcal{D}$ so that

$$
\widehat{L}_{U}\left(\tilde{f}_{0}\right) \leq \inf _{\phi \in \mathcal{D}} \widehat{L}_{U}(\phi)+\varepsilon
$$

Step 2 For $k=1, \ldots, M-1$, let

$$
\tilde{f}_{k}=u\left(\left(1-\pi_{k}\right) \xi\left(\tilde{f}_{k-1}\right)+\pi_{k} \xi(\tilde{\phi})\right),
$$

where $\tilde{\phi} \in \mathcal{D}$ is chosen so that

$$
\widehat{L}_{U}\left(\tilde{f}_{k}\right) \leq \inf _{\phi \in \mathcal{D}} \widehat{L}_{U}\left(u\left(\left(1-\pi_{k}\right) \xi\left(\tilde{f}_{k-1}\right)+\pi_{k} \xi(\phi)\right)\right)+\pi_{k} \varepsilon .
$$

Step 3 Let $\widehat{f}=\tilde{f}_{M-1}$.

The final form of $\widehat{f}$ can be expressed as

$$
\widehat{f}(x)=u\left(\sum_{\ell=0}^{M-1} q_{\ell} \xi\left(\tilde{\phi}_{\ell}(x)\right)\right)
$$

where $q_{\ell}(\ell=0,1, \ldots, M-1)$ is the set of mixing coefficients obtained as

$$
q_{\ell}=\pi_{\ell} \prod_{t=\ell+1}^{M-1}\left(1-\pi_{t}\right)
$$

with $\pi_{0}=1$ (see Remark 2 in Klemelä 2007), and each $\tilde{\phi}_{\ell}$ is the word in $\mathcal{D}$ selected during each iteration of Step 2 of the algorithm. An optimal word $\tilde{\phi}_{\ell}$ satisfying the condition in Step 2 can always be found since $\mathcal{D}$ is a finite set. $\widehat{f}$ and $q_{\ell}$ can be derived recursively from the definition in the algorithm. It is easy to verify that $\sum_{\ell=0}^{M-1} q_{\ell}=1$, and hence $\widehat{f}$ can be 
seen as the image of a convex combination of elements in the transformed dictionary $\xi(\mathcal{D})$ by $u=\xi^{-1}$.

Note that $\widehat{f}$ is not always a bona fide density (Wand and Jones 1993): its integral is not 1. Hence, we may also consider its normalized form

$$
\widehat{f_{c}}(x)=\gamma^{-1} \widehat{f}(x), \quad \gamma=\int_{\mathbb{R}^{d}} \widehat{f}(x) d x .
$$

Another candidate for a density estimator is a simple mixture

$$
\bar{f}(x)=u\left(\sum_{\phi \in \mathcal{D}} \bar{\lambda}_{\phi} \xi(\phi(x))\right)
$$

which satisfies

$$
\widehat{L}_{U}(\bar{f}) \leq \inf _{g \in \operatorname{CO}(\xi(\mathcal{D}))} \widehat{L}_{U}(u(g))+\varepsilon
$$

That is, $\bar{f}$ is the estimator minimizing the empirical risk over $\operatorname{co}(\xi(\mathcal{D}))$. The difference between $\widehat{f}$ and $\bar{f}$ is whether the words $\phi \in \mathcal{D}$ accumulated to construct the density estimator are selected via the algorithm or not. The $\bar{f}$ has smaller error than $\widehat{f}$ as described in Sect. 4 . However we do not pursue the practical algorithm for obtaining $\bar{f}$, since it is not an easy task unless the number of components $k$ in the mixture is given and fixed.

\subsection{Interpretation}

Here we briefly discuss the interpretation of the algorithm. In Step 2 of the algorithm the empirical loss

$$
\widehat{L}_{U}\left(u\left(\left(1-\pi_{k}\right) \xi\left(\tilde{f}_{k-1}\right)+\pi_{k} \xi(\phi)\right)\right)
$$

is minimized on the dictionary $\mathcal{D}$. It is easy to confirm from (2) that the decomposition

$$
\begin{aligned}
& \widehat{L}_{U}\left(u\left(\left(1-\pi_{k}\right) \xi\left(\tilde{f}_{k-1}\right)+\pi_{k} \xi(\phi)\right)\right) \\
& \quad=\left(1-\pi_{k}\right) \widehat{L}_{U}\left(\tilde{f}_{k-1}\right)+\pi_{k} \widehat{L}_{U}(\phi)-\Delta_{\xi}\left(\pi_{k} \mid \tilde{f}_{k-1}, \phi\right)
\end{aligned}
$$

holds, where

$$
\begin{aligned}
\Delta_{\xi}(\pi \mid f, \phi)= & \int_{\mathbb{R}^{d}}[(1-\pi) U(\xi(f(x)))+\pi U(\xi(\phi(x))) \\
& -U((1-\pi) \xi(f(x))+\pi \xi(\phi(x)))] d x
\end{aligned}
$$

for $0<\pi<1$, which is nonnegative by the convex property of $U$. Hence, the minimization can be implemented only through the second and third terms of the right hand side of (4).

The minimization of $\pi_{k} \widehat{L}_{U}(\phi)$ as a function of $\phi$ is easy to understand. On the other hand, the minimization of $-\Delta_{\xi}\left(\pi_{k} \mid \tilde{f}_{k-1}, \phi\right)$ as a function of $\phi$ can be carried out by finding a $\phi$ which is as far from $\tilde{f}_{k-1}$ as possible. Therefore, the algorithm can be understood as attempting to find a $\phi$ which makes the empirical $U$-loss function as small as possible and is simultaneously as far from the current estimator $\tilde{f}_{k-1}$ as possible. 


\subsection{Related methods}

The form of $\widehat{f}$ and the algorithm described in Sect. 3.1 suggest a relationship between $\widehat{f}$ and other often-discussed methods.

Kernel density estimator Kernel density estimation is a useful tool for nonparametric density estimation, and has been widely discussed in the literature (Silverman 1986; Wand and Jones 1993; Simonoff 1996). It can be easily extended to a multivariate setting. Several sophisticated methods to select the smoothing parameter included in the estimator have been developed, so that the technique is now fully data-based (Jones et al. 1996; Duong and Hazelton 2003, 2005a, 2005b). We show here that $\widehat{f}$ can be seen as a variant of the kernel density estimator. Let the dictionary be given by

$$
\mathcal{D}_{K}=\left\{K_{H}\left(\cdot-X_{i}\right) \mid i=1, \ldots, n\right\},
$$

where $K$ is a kernel function which is typically a symmetric $d$-variate density function, $H$ is a positive definite $d \times d$ bandwidth matrix and $K_{H}(z)=\left|H^{-1 / 2}\right| K\left(H^{-1 / 2} z\right)$ for $z \in \mathbb{R}^{d}$. Consider the case $\beta=1$ in $\beta$-power divergence. Then the finally obtained estimator has the form

$$
\widehat{f}(x)=\widehat{f}_{\mathrm{KDE}}(x)=u\left(\sum_{\ell=0}^{M-1} q_{\ell} \xi\left(\tilde{\phi}_{\ell}(x)\right)\right)=\sum_{\ell=0}^{M-1} q_{\ell} \tilde{\phi}_{\ell}(x) .
$$

Here we note that each $\tilde{\phi}_{\ell}$ is selected from $\mathcal{D}_{K}$. We consider a map

$$
\lambda:\{0,1, \ldots, M-1\} \rightarrow\{1, \ldots, n\}
$$

defined as $\lambda(\ell)=i$, if $\tilde{\phi}_{\ell}(x)=K_{H}\left(x-X_{i}\right)$. Furthermore, we define

$$
w_{\lambda}(i)=\left\{\begin{array}{cl}
\sum_{\ell=0}^{M-1} 1\left\{\ell \in \lambda^{-1}(\{i\})\right\} q_{\ell}, & \lambda^{-1}(\{i\}) \neq \text { empty } \\
0, & \lambda^{-1}(\{i\})=\text { empty },
\end{array}\right.
$$

for $i=1, \ldots, n$. Using these we have

$$
\widehat{f}_{\mathrm{KDE}}(x)=\sum_{i=1}^{n} w_{\lambda}(i) K_{H}\left(x-X_{i}\right),
$$

which can be seen as a weighted form of the kernel density estimator. The usual kernel density estimator can be understood as a special case of $w_{\lambda}(i)=n^{-1}$ for $i=1, \ldots, n$.

Boosting As a useful and effective learning method, boosting has been investigated in the field of statistical learning. Its aim is to construct a powerful committee by combining weak learners (words) in a suitable way. Many applications of boosting to pattern recognition have been developed, as summarized by Hastie et al. (2009). Boosting has also been shown to perform well in the field of regression (Bühlman and Yu 2003). Application of the concept of boosting to density estimation has been discussed by Ridgeway (2002) and Rosset and Segal (2002). In the boosting algorithm, the mixing coefficient $\pi_{k}$ is optimized using a line search. Apart from the use of this line search, the algorithm described earlier in this section is almost identical to a boosting algorithm. A clear explanation on the relationship between boosting and the empirical minimization algorithm in the case of $\beta$-power divergence with $\beta=1$ can be found in Klemelä (2007). An interesting combination of boosting and kernel density estimation was discussed by Di Marzio and Taylor (2004). 


\section{Error bounds}

In this section we evaluate theoretical performance of the proposal $\widehat{f}$ and its normalized form $\widehat{f}_{c}$. The result for a simple mixture $\bar{f}$ is also given. All theoretical results will be derived without any asymptotic manipulations, and hence they are non-asymptotic results. The results include those reported by Klemelä (2007) as special cases.

\subsection{Assumptions}

Throughout this paper, we utilize

$$
\pi_{k}=\frac{\theta}{k+\theta}, \quad k=1, \ldots, M-1
$$

with $\theta \geq 2$ for the mixing coefficients included in $\widehat{f}$ and define $\pi_{0}=1$. Note that $\theta=2$ was used in Klemelä (2007).

We consider a triplet

$$
\Phi=\left(\sum_{m=1}^{T} q_{m} \xi\left(\tilde{\phi}_{m}\right), \phi, \bar{\phi}\right), \quad \sum_{m=1}^{T} q_{m} \xi\left(\tilde{\phi}_{m}\right) \in \operatorname{co}(\xi(\mathcal{D})), \phi, \bar{\phi} \in \mathcal{D} .
$$

The collection of these triplets is denoted by

$$
\begin{aligned}
H(\mathcal{D}) & \equiv \operatorname{co}(\xi(\mathcal{D})) \times \mathcal{D} \times \mathcal{D} \\
& =\left\{\Phi=\left(\sum_{m=1}^{T} q_{m} \xi\left(\tilde{\phi}_{m}\right), \phi, \bar{\phi}\right) \mid \sum_{m=1}^{T} q_{m} \xi\left(\tilde{\phi}_{m}\right) \in \operatorname{co}(\xi(\mathcal{D})), \phi, \bar{\phi} \in \mathcal{D}\right\} .
\end{aligned}
$$

For $\delta \in[0,1]$ and $\Phi \in H(\mathcal{D})$, we define

$$
\begin{aligned}
& \psi_{U}(\delta \mid \Phi) \\
& \quad=\int_{\mathbb{R}^{d}} U^{\prime \prime}\left((1-\delta) \sum_{m=1}^{T} q_{m} \xi\left(\tilde{\phi}_{m}(x)\right)+\delta \xi(\phi(x))\right)\{\xi(\phi(x))-\xi(\bar{\phi}(x))\}^{2} d x .
\end{aligned}
$$

We require the following assumptions to establish the theoretical results:

Assumption 1 There exists a constant $B_{U}^{2}>0$ such that

$$
\sup _{\delta \in[0,1]} \sup _{\Phi \in H(\mathcal{D})} \psi_{U}(\delta \mid \Phi) \leq B_{U}^{2}
$$

Assumption 2 There exist two constants $C_{U}>0$ and $0<\alpha \leq 1$ such that

$$
\inf _{\delta \in[0,1]} U^{\prime \prime}\left(\xi\left((1-\delta) \bar{f}(x)+\delta \bar{f}_{c}(x)\right)\right) \geq C_{U} \bar{f}(x)^{\alpha}>0
$$

for any $x \in \mathbb{R}^{d}$ and for any $\bar{f}=u\left(\sum_{m=1}^{T} q_{m} \xi\left(\tilde{\phi}_{m}\right)\right)$ with $\sum_{m=1}^{T} q_{m} \xi\left(\tilde{\phi}_{m}\right) \in \operatorname{co}(\xi(\mathcal{D}))$, where $\bar{f}_{c}$ is the normalized form of $\bar{f}$. 
Example 3 (The Kullback-Leibler divergence) We introduce a constant $B_{K L}^{2}$ defined as

$$
B_{K L}^{2}=\sup _{\phi, \bar{\phi}, \tilde{\phi} \in \mathcal{D}} \int_{\mathbb{R}^{d}} \tilde{\phi}(x)\{\log \phi(x)-\log \bar{\phi}(x)\}^{2} d x .
$$

Then we see that

$$
\psi_{U}(\delta \mid \Phi) \leq B_{K L}^{2},
$$

for any $\delta \in[0,1]$ and any $\Phi \in H(\mathcal{D})$ in the case of Kullback-Leibler divergence. For the constants in Assumption 2, we may choose $\alpha=1$ and $C_{U}=\min \left\{1, \bar{\gamma}^{-1}\right\}$ for the KullbackLeibler divergence, where

$$
\bar{\gamma}=\int_{\mathbb{R}^{d}} \bar{f}(x) d x
$$

see Appendix A for the proof.

Example 4 ( $\beta$-power divergence) The constant $B_{U}^{2}$ for $\beta$-power divergence is obtained as

$$
B_{\beta}^{2}=\sup _{g \in \operatorname{CO}(\mathcal{D})} \sup _{\phi, \bar{\phi} \in \mathcal{D}} \frac{1}{\beta^{2}} \int_{\mathbb{R}^{d}} g(x)^{1-\beta}\left\{\phi(x)^{\beta}-\bar{\phi}(x)^{\beta}\right\}^{2} d x .
$$

Furthermore, $\alpha=1-\beta$ and $C_{U}=\min \left\{1, \bar{\gamma}^{\beta-1}\right\}$ can be chosen for $\beta$-power divergence. These are verified in Appendix A.

Examples 3-4 reveal that neither of our assumptions are very restrictive if we utilize a reasonable dictionary. Specifically, we can show that both constants $B_{K L}^{2}$ and $B_{\beta}^{2}$ are bounded if a certain set of Gaussian densities is used as the dictionary.

\subsection{Lemmas}

We use the following notation for convex combination:

$$
f(x, \Lambda)=f(x, \Lambda, \mathcal{D})=u\left(\sum_{\phi \in \mathcal{D}} \lambda_{\phi} \xi(\phi(x))\right), \quad x \in \mathbb{R}^{d},
$$

where $\Lambda=\left(\lambda_{\phi}\right)_{\phi \in \mathcal{D}} \in \mathcal{W}$, and $\mathcal{W}$ is the set of vectors of coefficients of finite convex combinations, expressed as

$$
\mathcal{W}=\left\{\left(\lambda_{\phi}\right)_{\phi \in \mathcal{D}} \mid \lambda_{\phi} \geq 0, \sum_{\phi \in \mathcal{D}} \lambda_{\phi}=1, \#\left\{\lambda_{\phi}>0\right\}<\infty\right\} .
$$

Note that $\{f(\cdot, \Lambda) \mid \Lambda \in \mathcal{W}\}=u(\operatorname{co}(\xi(\mathcal{D})))$.

We next introduce two functions which play important roles in theoretical evaluation in Lemma 4 . Let $\tilde{f}$ be any density estimator and let

$$
f^{*}(x)=u\left(\sum_{j=1}^{N} p_{j} \xi\left(\phi_{j}(x)\right)\right)
$$

where $p_{j} \geq 0, \sum_{j=1}^{N} p_{j}=1$, and $\phi_{j} \in \mathcal{D}$. 
We consider two functions of the variable $\pi \in[0,1]$ defined as follows:

$$
\begin{aligned}
\theta\left(\pi \mid \tilde{f}, f^{*}\right)= & \sum_{j=1}^{N} p_{j} \int_{\mathbb{R}^{d}}\left[U\left((1-\pi) \xi(\tilde{f}(x))+\pi \xi\left(\phi_{j}(x)\right)\right)\right. \\
& \left.-(1-\pi) U(\xi(\tilde{f}(x)))-\pi U\left(\xi\left(f^{*}(x)\right)\right)\right] d x, \\
\eta\left(\pi \mid \tilde{f}, f^{*}\right)= & \sum_{j=1}^{N} p_{j} \int_{\mathbb{R}^{d}}\left[U\left((1-\pi) \xi(\tilde{f}(x))+\pi \xi\left(\phi_{j}(x)\right)\right)\right. \\
& \left.-U\left((1-\pi) \xi(\tilde{f}(x))+\pi \xi\left(f^{*}(x)\right)\right)\right] d x .
\end{aligned}
$$

For these two functions $\theta(\pi) \equiv \theta\left(\pi \mid \tilde{f}, f^{*}\right)$ and $\eta(\pi) \equiv \eta\left(\pi \mid \tilde{f}, f^{*}\right)$, we can verify the following properties:

Lemma 1 Let $\tilde{f}$ be a density estimator of $f$ and let $f^{*}$ be the estimator given in (7). For $0 \leq \pi \leq 1$, it then follows that

$$
\begin{aligned}
& \sum_{j=1}^{N} p_{j} \widehat{L}_{U}\left(u\left((1-\pi) \xi(\tilde{f})+\pi \xi\left(\phi_{j}\right)\right)\right)-\widehat{L}_{U}\left(f^{*}\right) \\
& =(1-\pi)\left[\widehat{L}_{U}(\tilde{f})-\widehat{L}_{U}\left(f^{*}\right)\right]+\theta\left(\pi \mid \tilde{f}, f^{*}\right)
\end{aligned}
$$

Lemma 2 Let $\tilde{f}$ be a density estimator of $f$ and let $f^{*}$ be the estimator given in (7). Then for any $\pi \in[0,1]$,

$$
\theta(\pi)=\theta\left(\pi \mid \tilde{f}, f^{*}\right) \leq \eta\left(\pi \mid \tilde{f}, f^{*}\right)=\eta(\pi)
$$

Lemma 3 Let $f^{*}$ be as given in (7) and let

$$
\tilde{f}=u\left(\sum_{\ell=1}^{k} q_{\ell} \xi\left(\tilde{\phi}_{\ell}\right)\right)
$$

for some $\sum_{\ell=1}^{k} q_{\ell} \xi\left(\tilde{\phi}_{\ell}\right) \in \operatorname{co}(\xi(\mathcal{D}))$. Under Assumption 1, it follows that

$$
\eta(\pi)=\eta\left(\pi \mid \tilde{f}, f^{*}\right) \leq \pi^{2} B_{U}^{2} \quad \text { for any } \pi \in[0,1] .
$$

Remark 1 In Lemmas 1 and 2, $\tilde{f}$ is assumed to be any density estimator. On the other hand, in Lemma 3, it is assumed to be an element of $u(\operatorname{co}(\xi(\mathcal{D})))$. By combining Lemma 3 with Lemma 2, we see that $\pi^{2} B_{U}^{2}$ is an upper bound of $\theta\left(\pi \mid \tilde{f}, f^{*}\right)$. It will be shown in the Appendix that getting the upper bound as "the square of $\pi$ " is crucial for obtaining Lemma 4 which gives the bound for the $U$-loss of the density estimator. For the case of the $L_{2}$ norm, an upper bound of $\theta\left(\pi \mid \tilde{f}, f^{*}\right)$ can be found by a straightforward evaluation, which is a constant times $\pi^{2}$, see Klemelä (2007). On the other hand, direct evaluation of $\theta\left(\pi \mid \tilde{f}, f^{*}\right)$ was not easy for the case of $U$-divergence. To overcome the difficulty, we introduced $\eta\left(\pi \mid \tilde{f}, f^{*}\right)$, of which an upper bound can be obtained as $\pi^{2} B_{U}^{2}$ as in Lemma 3. In this sense, Lemma 3 represents an important contribution to the generalization of the results based on the $L_{2}$ norm discussed in Klemelä (2007) to those based on the $U$-divergence. 
Lemma 4 For stagewise minimization density estimator $\widehat{f}$, it holds under Assumption 1 that

$$
\widehat{L}_{U}(\widehat{f}) \leq \inf _{\Lambda \in \mathcal{W}} \widehat{L}_{U}(f(\cdot, \Lambda))+\frac{\theta^{2} B_{U}^{2}}{M+(\theta-1)}+\varepsilon
$$

Lemma 5 For $\tau>0$, let $\hat{f} \in\{f(\cdot, \Lambda) \mid \Lambda \in \mathcal{W}\}$ be such that

$$
\widehat{L}_{U}(\hat{f}) \leq \inf _{\Lambda \in \mathcal{W}} \widehat{L}_{U}(f(\cdot, \Lambda))+\tau \text {. }
$$

Then

$$
D_{U}(f, \hat{f}) \leq \inf _{\Lambda \in \mathcal{W}} D_{U}(f, f(\cdot, \Lambda))+\tau+2 \sup _{\phi \in \mathcal{D}}\left|v_{n}(\xi(\phi))\right|
$$

Lemma 6 For any $h \in \operatorname{co}(\xi(\mathcal{D}))$, let $g(x)=u(h(x))$ and let $g_{c}(x)=v_{g}^{-1} g(x)$, where

$$
v_{g}=\int_{\mathbb{R}^{d}} g(x) d x .
$$

Under Assumption 2, we have

$$
D_{U}\left(f, g_{c}\right) \leq D_{U}(f, g)+C_{U}^{-1}\left|1-v_{g}^{-1}\right| \int_{\mathbb{R}^{d}}\left|g_{c}(x)-f(x)\right| g(x)^{1-\alpha} d x .
$$

4.3 Error bound for stagewise estimator

A non-asymptotic error bound for $\widehat{f}$ can be summarized as follows:

Theorem 1 For the density estimator $\widehat{f}$, it holds under Assumption 1 that

$$
E_{f} D_{U}(f, \widehat{f}) \leq \inf _{g \in \operatorname{co}(\xi(\mathcal{D}))} D_{U}(f, u(g))+2 E_{f} \sup _{\phi \in \mathcal{D}}\left|v_{n}(\xi(\phi))\right|+\frac{\theta^{2} B_{U}^{2}}{M+(\theta-1)}+\varepsilon,
$$

where $v_{n}(\cdot)$ is the centered empirical operator defined by

$$
v_{n}(\xi(\phi))=\frac{1}{n} \sum_{i=1}^{n} \xi\left(\phi\left(X_{i}\right)\right)-\int_{\mathbb{R}^{d}} \xi(\phi(x)) f(x) d x .
$$

Remark 2 The term $\inf _{g \in \operatorname{co}(\xi(\mathcal{D}))} D_{U}(f, u(g))$ can be seen as the essential bias in approximating $f$ by $u(g), g \in \operatorname{co}(\xi(\mathcal{D}))$. The main variation is captured in the term $E_{f} \sup _{\phi \in \mathcal{D}}\left|v_{n}(\xi(\phi))\right|$, which can be interpreted as the variance. We observe that these terms are natural generalizations of the corresponding terms in Theorem 1 of Klemelä (2007) in which a non-asymptotic error bound for the estimator based on the $L_{2}$ norm ( $\beta$-divergence with $\beta=1$ ) is given.

Remark 3 The case $\beta=1$ in $\beta$-power divergence produces a half of squared $L_{2}$ norm, which means that twice the error bound in the right hand side of Theorem 1 should coincide with the error bound given in Theorem 1 of Klemelä (2007). However, the term including $\theta$ is actually not the same as the corresponding term in Theorem 1 of Klemelä (2007), since the 
value $\theta=2$ utilized in Klemelä (2007) yields a constant $2 \theta^{2}=8$ but a constant 4 appears in Klemelä (2007). This reveals that the $L_{2}$ norm can provide a sharper bound; see the proof of Lemma 3 in Klemelä (2007).

Remark 4 It might be possible to obtain a simplified expression of the error bound in Theorem 1 provided that we make some appropriate assumptions about the utilized dictionary $\mathcal{D}$. Discussion on this topic can be found in Klemelä (2007) for the $L_{2}$ norm estimator, although the simplification itself is not pursued in this paper. We note that the error bound is expected to be small if the iteration $M$ grows.

Theorem 2 For the normalized form $\widehat{f_{c}}$ of $\widehat{f}$, it follows under Assumptions 1 and 2 that

$$
E_{f} D_{U}\left(f, \widehat{f_{c}}\right) \leq E_{f} D_{U}(f, \widehat{f})+C_{U}^{-1} E_{f}\left[\left|1-c^{-1}\right| \int_{\mathbb{R}^{d}}\left|\widehat{f_{c}}(x)-f(x)\right| \widehat{f}(x)^{1-\alpha} d x\right] .
$$

Remark 5 Theorem 2 reveals that the bound for the normalized estimator $\widehat{f_{c}}$ corresponds to the bound for $\widehat{f}$ plus an extra term which is the effect of involving a stochastic normalized constant $c$.

\subsection{Error bound for mixture estimator}

The mixture estimator has a smaller risk than the stagewise estimator:

Theorem 3 For the mixture estimator $\bar{f}$ in (3), we have

$$
E_{f} D_{U}(f, \bar{f}) \leq \inf _{g \in \operatorname{co}(\xi(\mathcal{D}))} D_{U}(f, u(g))+2 E_{f} \sup _{\phi \in \mathcal{D}}\left|v_{n}(\xi(\phi))\right|+\varepsilon .
$$

As we can see from the definition, $\bar{f}$ cannot be realistically determined in practice. In this sense, Theorem 3 is just an ideal result in a purely theoretical consideration. However, we can see from the proofs given in the Appendix that Theorem 3 is crucial to obtain Theorem 1.

\section{Simulation}

The practical performance of the stagewise minimization density estimator was investigated by simulation in a bivariate situation. A comparison of the proposed estimator with existing kernel-type density estimators was also implemented. Besides the fact that the bivariate situation is important in its own right, we chose it for simplicity and in the hope that the lessons learned are applicable to higher dimensions.

\subsection{Simulation setup}

As true target densities $f$, the bivariate normal mixture densities utilized in Wand and Jones (1993) were adopted. In particular, Case C (Skewed - Unimodal), Case H (Bimodal IV), and Case L (Quadrimodal) were used.

The utilized dictionary was a family of Gaussian densities defined as

$$
\mathcal{D}=\left\{\phi_{\Sigma}\left(x-X_{i}\right) \mid i=1, \ldots, n, \Sigma \in \mathcal{S}\right\},
$$


where $\phi_{V}(x-\mu)$ is the density of $N_{d}(\mu, V)$ and $\mathcal{S}$ is a set of covariance matrices given as

$$
\begin{gathered}
\mathcal{S}=\left\{\left[\begin{array}{ll}
1 & 0 \\
0 & 1
\end{array}\right],\left[\begin{array}{ll}
\frac{1}{4} & 0 \\
0 & 1
\end{array}\right],\left[\begin{array}{ll}
1 & 0 \\
0 & \frac{1}{4}
\end{array}\right],\left[\begin{array}{cc}
1 & \frac{1}{2} \\
\frac{1}{2} & 1
\end{array}\right],\left[\begin{array}{cc}
1 & -\frac{1}{2} \\
-\frac{1}{2} & 1
\end{array}\right],\right. \\
\left.\left[\begin{array}{ll}
1 & 1 \\
1 & 4
\end{array}\right],\left[\begin{array}{cc}
\frac{4}{9} & -\frac{28}{90} \\
-\frac{28}{90} & \frac{4}{9}
\end{array}\right],\left[\begin{array}{cc}
\frac{4}{9} & \frac{12}{45} \\
\frac{12}{45} & \frac{4}{9}
\end{array}\right],\left[\begin{array}{cc}
\frac{9}{25} & -\frac{9}{50} \\
-\frac{9}{50} & \frac{9}{25}
\end{array}\right],\left[\begin{array}{cc}
\frac{9}{25} & \frac{9}{50} \\
\frac{9}{50} & \frac{9}{25}
\end{array}\right]\right\} .
\end{gathered}
$$

The performance of the estimators minimizing the $\beta$-power divergence with $\beta=1$ (usual $L_{2}$ ) and $\beta=0.5$ was investigated, where these estimators are expressed as $\widehat{f_{1}}$ and $\widehat{f_{0.5}}$, respectively. Actually, $\widehat{f_{0.5}}$ designates the normalized form. The sample size was set to $n=$ 50,200 and the number of iterations of the algorithm for $\widehat{f_{0.5}}$ and $\widehat{f_{1}}$ was $M=50$.

The Mean Integrated Squared Error (MISE) defined as

$$
\operatorname{MISE}[\widehat{f}]=E \int_{\mathbb{R}^{2}}\{\widehat{f}(x)-f(x)\}^{2} d x
$$

was used as the accuracy measure, which is nothing other than twice the value of $E D_{\beta}(f, \widehat{f})$ with $\beta=1$.

We adopted the kernel density estimator $\widehat{f}_{\mathrm{KDE}}$ in Sect. 3.4 with a Gaussian kernel and the direct plug-in bandwidth matrix $H$ proposed by Duong and Hazelton (2003) as a competitor, which is easily implemented using a package " $k s$ " in R (see Duong 2010).

As an another kernel-type competitor, the reduced set density estimator (RSDE) proposed in Girolami and He (2003) was also demonstrated in simulation. The RSDE, denoted by $\widehat{f_{\text {RSDE}}}$, has the form

$$
\widehat{f}_{\mathrm{RSDE}}(x)=\sum_{i=1}^{n} a(i) K_{b}\left(x-X_{i}\right),
$$

where $a(i) \geq 0(i=1, \ldots, n)$ satisfying $\sum_{i=1}^{n} a(i)=1, b$ is a scalar bandwidth determined by cross-validation. Determining efficient weight $a(i) \mathrm{s}$ in conjunction with sparseness is a main purpose of the algorithm for RSDE. Note that $\widehat{f}_{\mathrm{KDE}}$ also has the similar form with $a(i)=n^{-1}$ and an efficient bandwidth matrix. In this regard, $\widehat{f}_{\mathrm{KDE}}$ aims to estimate $f$ by using a simple weight $a(i)$ and a sophisticated bandwidth. On the other hand, $\widehat{f}_{\mathrm{RSDE}}$ tries to capture the density structure with an efficiently determined weights $a(i) \mathrm{s}$, though the bandwidth $b$ is simply selected.

Note that we do not need to approximate ISE for all estimators simulated here, since all estimators are with Gaussian kernels and hence calculation of the integrals can be carried out exactly by the repeated use of the properties of Gaussian densities summarized in Wand and Jones (1993, Appendix C). The normalized constant for $\widehat{f_{0.5}}$ can also be precisely calculated.

\subsection{Results}

A set of data of size $n$ was generated from $f$, and (1/2)ISE for $\widehat{f_{0.5}}$ and $\widehat{f_{1}}$ were calculated at each iteration step $M$ up to 50. Using the same data set, (1/2)ISE for $\widehat{f}_{\mathrm{KDE}}$ and $\widehat{\mathrm{P}}_{\mathrm{PSDE}}$ (PSDE) were also calculated. Ten simulations were carried out, and then, for each estimator, we calculated an estimate of (1/2)MISE based as the mean of ten (1/2)ISE values obtained by simulation. The estimates of (1/2)MISE are shown in Figs. 1-3. 
(A) $n=50$

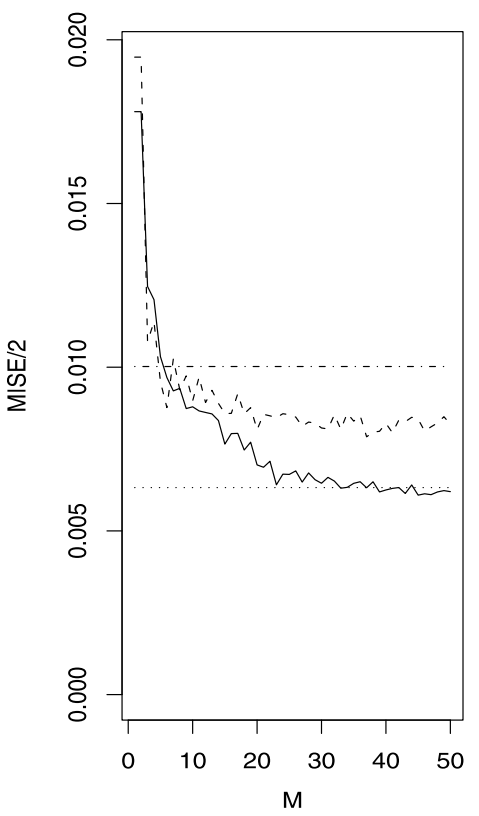

(B) $n=200$

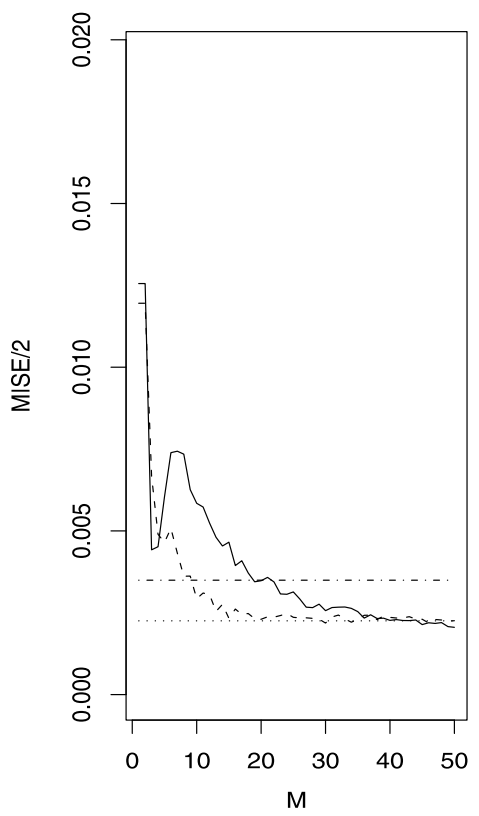

Fig. 1 Results of simulation for Case C. Panel (A) is for $n=50$ and panel (B) is for $n=200$. Solid line corresponds to $(1 / 2) \mathrm{MISE}$ for $\widehat{f}_{0.5}$; dashed line is for $\widehat{f}_{1}$; dashed-dotted line is for $\widehat{f}_{\mathrm{KDE}}$; dotted line is for $\widehat{f}_{\mathrm{RSDE}}$. dashed-dotted line and dotted lines are both flat because no iteration occurs. Standard error for all estimators are less than $11 \times 10^{-4}$ for $n=50$ and less than $5 \times 10^{-4}$ for $n=200$

The results for Case $\mathrm{C}$ are displayed in Fig. 1. It can be seen from panel (A) that $\widehat{f}_{0.5}$ and $\widehat{f_{1}}$ both behave better than $\widehat{f}_{\mathrm{KDE}}$. The $\widehat{f}_{\mathrm{RSDE}}$ performs totally well, however $\widehat{f}_{0.5}$ becomes to be marginally better than $\widehat{f}_{\mathrm{RSDE}}$ when the iteration $M$ exceeds 40 .

The almost same tendency can be observed in the panel (B): $\widehat{f}_{\mathrm{RSDE}}$ performs well, $\widehat{f}_{1}$ and $\widehat{f_{0.5}}$ are better than $\widehat{f_{\mathrm{KDE}}}$. The $\widehat{f_{1}}$ is competitive to $\widehat{f}_{\mathrm{RSDE}}$ in a early stage of iteration. And $\widehat{f}_{0.5}$ becomes finally to be a bit superior to $\widehat{f}_{\mathrm{RSDE}}$ for $M \geq 45$. This good performance of $\widehat{f}_{0.5}$ appears to validate the use of $\beta$ values less than $1(0<\beta<1)$ in $\beta$-power divergence.

Figure 2 shows the results for Case $\mathrm{H}$ corresponding to a bimodal density in which each component has a different correlation structure. We observe in the panel (A) that the performances of $\widehat{f}_{0.5}$ and $\widehat{f}_{1}$ are both better than $\widehat{f}_{\mathrm{KDE}}$ and $\widehat{f}_{\mathrm{RSDE}}$ for iteration steps $M \geq 20$. Both $\widehat{f}_{\mathrm{KDE}}$ and $\widehat{f}_{\mathrm{RSDE}}$ stay in the middle between $\widehat{f}_{0.5}$ and $\widehat{f}_{1}$ for $n=200$ as seen in the panel (B).

Finally, Fig. 3 shows the results for Case L, corresponding to a quadrimodal density with slightly complex shape. In this case, $\widehat{f}_{\mathrm{KDE}}$ performs better than others. On the other hand, $\widehat{f}_{\text {RSDE }}$ is worst among simulated estimators. In the panel (A), $\widehat{f_{1}}$ stably better than $\widehat{f_{0.5}}$ and becomes to be competitive to $\widehat{f}_{\mathrm{KDE}}$ for $M \geq 40$. A similar trend can be seen in the panel (B), in which $\widehat{f_{1}}$ is slightly better than $\widehat{f_{\mathrm{KDE}}}$ for $M \geq 35$. Though the $\widehat{f_{0.5}}$ curve decreases, it remains above that of $\widehat{f}_{\mathrm{KDE}}$. The curve appears to be stable for $M \geq 40$, and hence further improvement of $\widehat{f_{0.5}}$ might not be achieved even for larger values of $M$.

Though this is just a simple simulation, the following conclusions can be made from this simulation study: The $\widehat{f}_{1}$ performs well in all cases simulated here, and it is better than $\widehat{f}_{\mathrm{KDE}}$ even for $n=50$ (Case C). The high performance of $\widehat{f_{1}}$ in Cases $\mathrm{H}$ and $\mathrm{L}$ suggests that $\beta=1$ in $\beta$-power divergence yields a flexible estimator. The RSDE $\widehat{f}_{\mathrm{RSDE}}$ also performs well, but 
(A) $n=50$

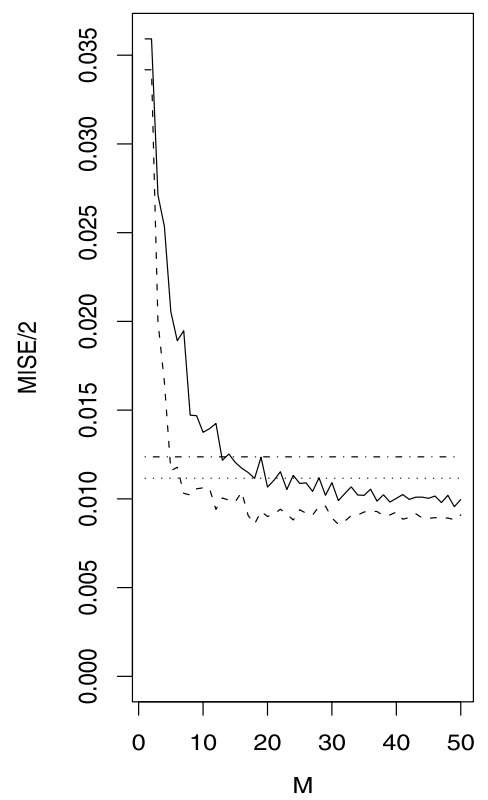

(B) $n=200$

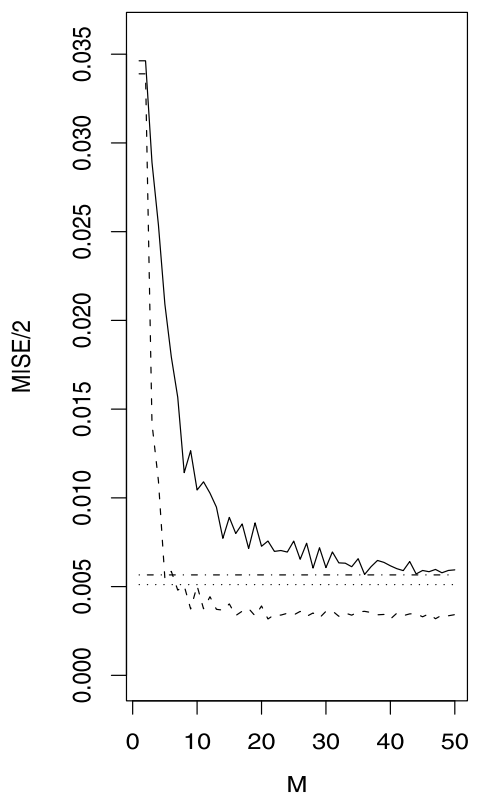

Fig. 2 Results of simulation for Case $\mathrm{H}$ for $n=200$. The lines are as described for Fig. 1. Standard error for all estimators are less than $15 \times 10^{-4}$ for $n=50$ and less than $10 \times 10^{-4}$ for $n=200$

it is a bit inferior to either $\widehat{f_{1}}$ or $\widehat{f_{0.5}}$ with a large iteration step $M$. The $\widehat{f_{\text {RSDE }}}$ might be good at estimating unimodal density as is observed in Case $\mathrm{C}$, however it does not guarantee a sufficient accuracy for multi-modal density as in Case L.

The $\widehat{f_{0.5}}$ performs well for unimodal density (Case C) and bimodal density with sufficiently separated components (Case H). It should be pointed out that (1/2)MISE corresponds to $\beta$-power divergence with $\beta=1$, and it is thus not a fair assessment of $\widehat{f_{0.5}}$ to utilize (1/2)MISE as the accuracy measure. It is noteworthy that $\widehat{f_{0.5}}$ with large $M$ is better than $\widehat{f_{1}}$ in Case $\mathrm{C}$, in spite of this unfavorable assessment. However, $\widehat{f_{0.5}}$ might not be good at estimating a multimodal density with crowded components as we observed in Case L. This might be related to the robustness property of the estimator for $\beta$-power divergence with $0<\beta<1$ (see Sect. 6).

\section{Discussion}

We proposed a method of stagewise minimization of the $U$-loss function in a unified manner to achieve good density estimation. The performance of the density estimator depends on the choice of $U$-loss function and the dictionary $\mathcal{D}$, as shown in Theorem 1 . It has previously been reported that a specific choice of the $U$-loss function led to robustness for parametric estimation in a classical model such as principal component analysis (Higuchi and Eguchi 2004) and an exponential family model (Fujisawa and Eguchi 2008). A related finding is that we have to choose $\mathcal{D}$ carefully in accordance with the selected $U$-loss function. For example, consider the $\beta$-power divergence as defined in Example 2. Then the resultant estimator takes 
(A) $n=50$

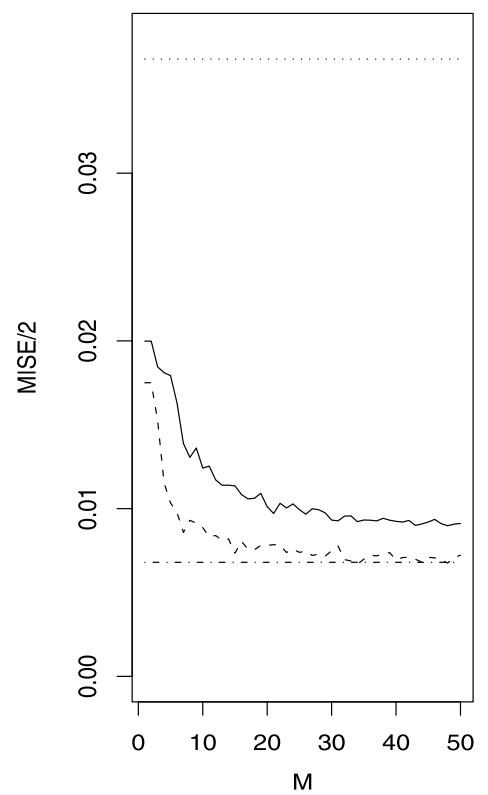

(B) $\mathbf{n}=\mathbf{2 0 0}$

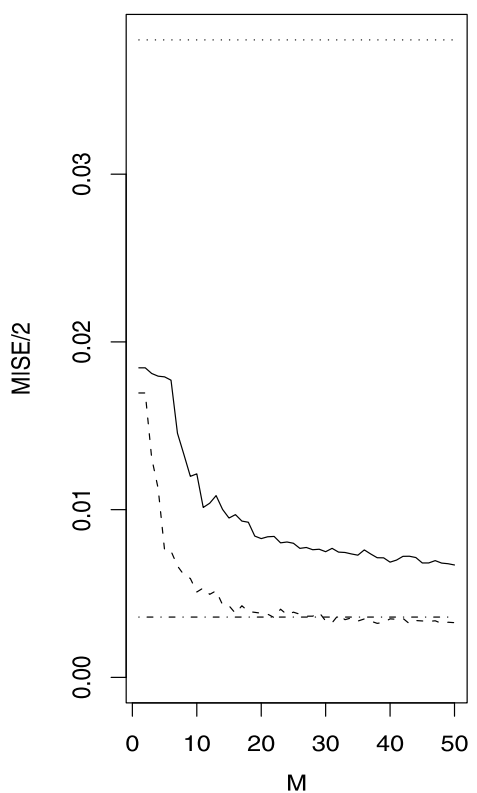

Fig. 3 Results of simulation for Case $\mathrm{L}$ for $n=200$. The lines are as described for Fig. 1. Standard error for all estimators are less than $30 \times 10^{-4}$ for $n=50$ and less than $12 \times 10^{-4}$ for $n=200$

the form

$$
\hat{f}_{\beta}(x)=\left\{\sum_{j=1}^{J} p_{j} \phi_{j}(x)^{\beta}\right\}^{\frac{1}{\beta}} .
$$

If we select Gaussian densities as $\phi_{j}(x)$ 's as in Sect. 5, the approximation performance becomes weaker as $\beta$ approaches 0 , since

$$
\lim _{\beta \rightarrow 0} \hat{f}_{\beta}(x)=\exp \left[\sum_{j=1}^{J} p_{j} \log \phi_{j}(x)\right] .
$$

In such a situation, the candidates in $\mathcal{D}$ should be chosen to be away from any exponential family model.

Selecting the dictionary $\mathcal{D}$ is an important issue, since the performance of estimators indeed depends on $\mathcal{D}$. From a theoretical view point, we might suggest, for example, to use $\mathcal{D}$ with finite $B_{\beta}^{2}$ in Example 4. It is not difficult to see that $B_{\beta}^{2}$ is finite provided that $\mathcal{D}$ consists of a finite set of Gaussian densities. The finiteness of $B_{\beta}^{2}$ is essentially depending on the existence of moments of densities, so that it might be possible to find another $\mathcal{D}$ with finite $B_{\beta}^{2}$.

From a practical view point, the dictionaries with Gaussian densities yield reasonable performance of density estimators, as seen in Sect. 5. In simulation study, we referred to some covariance matrices given in Table 1 of Wand and Jones (1993). Once the dictionary was fixed as Gaussian densities, our impression is that the class $\mathcal{S}$ utilized in this paper 
for covariance matrices is almost sufficient for estimating any shapes of bivariate densities. For higher dimensional cases, it is necessary to prepare covariance matrices with several correlation structures. For the mean, we used each data point in simulation, but this choice of mean depends on the sample size. Another candidate for the set of mean is to generate the grid points on the support of target density. This might be efficient, however it needs a rough estimate of the support region.

In this way, we can consider several ways to make a dictionary even if we restrict it as Gaussian densities. Although there does not seem to exist a lucid and conclusive way to select $\mathcal{D}$, it would be continually important to accumulate many experiments on its choice.

In this paper, our interest is confined to density estimation. However, this stagewise minimization method is applicable to many other forms of statistical analysis. For example, we can explore a finite Gaussian mixture by a sequential learning algorithm, as proposed in Sect. 3, if we adopt the set of all Gaussian densities as the dictionary. Basically, it is not necessary to fix the number of mixture components that can be selected in the sequential procedure. This is related to the $K$-means clustering and also with the mixture of principal component analysis models, the mixture of independent component analysis models and so forth. However, we have to carefully consider efficient regularization for the $U$-loss function because the dictionary $\mathcal{D}$ can prepare overly flexible words, which may lead to over-learning of the algorithm. This issue is a challenging subject for future work.

As was suggested in Sect. 1, not only density estimation but also statistical learning algorithms based on the $L_{2}$ norm may be generally extended to algorithms based on $U$ divergence. The work by Klemelä and Mammen (2010) approached inverse problems by utilizing the $L_{2}$ norm, and it might be interesting to envisage how their theory can be changed by using $U$-divergence.

Another generalization might be considered for a variant of the projection pursuit density estimation (PPDE) proposed by Friedman et al. (1984). In PPDE, a sequence of augmenting functions is determined via optimization of the (marginal) Kullback-Leibler divergence. Though the original PPDE is fully nonparametric, we can easily consider a variant of PPDE which is implemented through an algorithm similar to that in Sect. 3 of this paper with a suitable dictionary consisting of a set of (univariate) density functions on projected directions. Hence, it would be promising if non-asymptotic error bounds for a variant of PPDE could be established.

Acknowledgements The research was commenced when the first author visited The Institute of Statistical Mathematics. The first author would greatly acknowledge kind hospitality of the institute and financial support. The authors are grateful to an editor and three anonymous referees for their helpful comments.

\section{Appendix A: Proofs for results in examples}

Example 3 By referring to Example 1 and using convex property, we can verify for the Kullback-Leibler divergence that

$$
\begin{aligned}
\psi_{U}(\delta \mid \Phi)= & \int_{\mathbb{R}^{d}} \exp \left((1-\delta) \sum_{m=1}^{T} q_{m} \log \tilde{\phi}_{m}(x)+\delta \log \phi(x)\right) \\
& \times\{\log \phi(x)-\log \bar{\phi}(x)\}^{2} d x \\
\leq & \int_{\mathbb{R}^{d}}\left\{(1-\delta) \sum_{m=1}^{T} q_{m} \tilde{\phi}_{m}(x)+\delta \phi(x)\right\}\{\log \phi(x)-\log \bar{\phi}(x)\}^{2} d x
\end{aligned}
$$




$$
\begin{aligned}
= & (1-\delta) \sum_{m=1}^{T} q_{m} \int_{\mathbb{R}^{d}} \tilde{\phi}_{m}(x)\{\log \phi(x)-\log \bar{\phi}(x)\}^{2} d x \\
& +\delta \int_{\mathbb{R}^{d}} \phi(x)\{\log \phi(x)-\log \bar{\phi}(x)\}^{2} d x \\
\leq & (1-\delta) \sum_{m=1}^{T} q_{m} B_{K L}^{2}+\delta B_{K L}^{2} \\
= & B_{K L}^{2} .
\end{aligned}
$$

For constants in Assumption 2, by putting

$$
\bar{\gamma}=\int_{\mathbb{R}^{d}} \bar{f}(x) d x
$$

we see that

$$
\begin{aligned}
& U^{\prime \prime}\left(\xi\left((1-\delta) \bar{f}(x)+\delta \bar{f}_{c}(x)\right)\right) \\
& \quad=(1-\delta) \bar{f}(x)+\delta \bar{f}_{c}(x) \\
& \quad=(1-\delta) \bar{f}(x)+\delta \frac{1}{\bar{\gamma}} \bar{f}(x) \\
& \quad \geq \min \left\{1, \frac{1}{\bar{\gamma}}\right\} \bar{f}(x),
\end{aligned}
$$

from which we may choose $\alpha=1$ and $C_{U}=\min \left\{1, \bar{\gamma}^{-1}\right\}$ for the Kullback-Leibler divergence.

Example 4 First we have for $0<\beta \leq 1 / 2$ that

$$
\begin{aligned}
\psi_{U}(\delta \mid \Phi) & =\frac{1}{\beta^{2}} \int_{\mathbb{R}^{d}}\left\{(1-\delta) \sum_{m=1}^{T} q_{m} \tilde{\phi}_{m}(x)^{\beta}+\delta \phi(x)^{\beta}\right\}^{(1 / \beta)-1}\left\{\phi(x)^{\beta}-\bar{\phi}(x)^{\beta}\right\}^{2} d x \\
& =\frac{1}{\beta^{2}} \int_{\mathbb{R}^{d}}\left[\left\{(1-\delta) \sum_{m=1}^{T} q_{m} \tilde{\phi}_{m}(x)^{\beta}+\delta \phi(x)^{\beta}\right\}^{1 / \beta}\right]^{1-\beta}\left\{\phi(x)^{\beta}-\bar{\phi}(x)^{\beta}\right\}^{2} d x \\
& \leq \frac{1}{\beta^{2}} \int_{\mathbb{R}^{d}}\left[(1-\delta)\left\{\sum_{m=1}^{T} q_{m} \tilde{\phi}_{m}(x)^{\beta}\right\}^{1 / \beta}+\delta \phi(x)\right]^{1-\beta}\left\{\phi(x)^{\beta}-\bar{\phi}(x)^{\beta}\right\}^{2} d x \\
& \leq \frac{1}{\beta^{2}} \int_{\mathbb{R}^{d}}\left[(1-\delta) \sum_{m=1}^{T} q_{m} \tilde{\phi}_{m}(x)+\delta \phi(x)\right]^{1-\beta}\left\{\phi(x)^{\beta}-\bar{\phi}(x)^{\beta}\right\}^{2} d x \\
& \leq B_{\beta}^{2},
\end{aligned}
$$


where we have used the convex property of $z^{1 / \beta}$ for $0<\beta \leq 1 / 2$. On the other hand, using the concave property of $z^{\beta}$ for $1 / 2<\beta \leq 1$, we see that

$$
\begin{aligned}
\psi_{U}(\delta \mid \Phi) & =\frac{1}{\beta^{2}} \int_{\mathbb{R}^{d}}\left\{(1-\delta) \sum_{m=1}^{T} q_{m} \tilde{\phi}_{m}(x)^{\beta}+\delta \phi(x)^{\beta}\right\}^{(1 / \beta)-1}\left\{\phi(x)^{\beta}-\bar{\phi}(x)^{\beta}\right\}^{2} d x \\
& \leq \frac{1}{\beta^{2}} \int_{\mathbb{R}^{d}}\left[\left\{(1-\delta) \sum_{m=1}^{T} q_{m} \tilde{\phi}_{m}(x)+\delta \phi(x)\right\}^{\beta}\right]^{(1 / \beta)-1}\left\{\phi(x)^{\beta}-\bar{\phi}(x)^{\beta}\right\}^{2} d x \\
& =\frac{1}{\beta^{2}} \int_{\mathbb{R}^{d}}\left\{(1-\delta) \sum_{m=1}^{T} q_{m} \tilde{\phi}_{m}(x)+\delta \phi(x)\right\}^{1-\beta}\left\{\phi(x)^{\beta}-\bar{\phi}(x)^{\beta}\right\}^{2} d x \\
& \leq B_{\beta}^{2} .
\end{aligned}
$$

Hence we have $\psi_{U}(\delta \mid \Phi) \leq B_{\beta}^{2}$ for the $\beta$-power divergence. Furthermore, similar calculation as in Example 3 gives

$$
\begin{aligned}
& U^{\prime \prime}\left(\xi\left((1-\delta) \bar{f}(x)+\delta \bar{f}_{c}(x)\right)\right) \\
& \quad=\left\{(1-\delta) \bar{f}(x)+\delta \bar{f}_{c}(x)\right\}^{1-\beta} \\
& \quad \geq(1-\delta) \bar{f}(x)^{1-\beta}+\delta \bar{f}_{c}(x)^{1-\beta} \\
& \quad \geq \min \left\{1,\left(\frac{1}{\bar{\gamma}}\right)^{1-\beta}\right\} \bar{f}(x)^{1-\beta} .
\end{aligned}
$$

This shows that $\alpha=1-\beta$ and $C_{U}=\min \left\{1, \bar{\gamma}^{\beta-1}\right\}$ can be chosen for $\beta$-power divergence.

\section{Appendix B: Proofs of lemmas}

Proof of Lemma 1 In this proof of Lemma 1, abbreviation for integral by $x$ on $\mathbb{R}^{d}$ will be used to save space, for example $\int_{\mathbb{R}^{d}} g(x) d x$ will be denoted by $\int_{\mathbb{R}^{d}} g$ and $\int_{\mathbb{R}^{d}} U(\xi(g))$ means $\int_{\mathbb{R}^{d}} U(\xi(g(x))) d x$.

For any nonnegative functions $F$ and $G$, we note from (2) that

$$
\widehat{L}_{U}(u(F+G))=\widehat{L}_{U}(u(F))+\widehat{L}_{U}(u(G))+\int_{\mathbb{R}^{d}}\{U(F+G)-U(F)-U(G)\} .
$$

By repeated use of this property, we have

$$
\begin{aligned}
& \sum_{j=1}^{N} p_{j} \widehat{L}_{U}\left(u\left((1-\pi) \xi(\tilde{f})+\pi \xi\left(\phi_{j}\right)\right)\right)-\widehat{L}_{U}\left(f^{*}\right) \\
& =\sum_{j=1}^{N} p_{j}\left[\widehat{L}_{U}\left(u\left((1-\pi) \xi(\tilde{f})+\pi \xi\left(\phi_{j}\right)-\xi\left(f^{*}\right)\right)\right)+\widehat{L}_{U}\left(f^{*}\right)\right. \\
& \quad+\int_{\mathbb{R}^{d}} U\left((1-\pi) \xi(\tilde{f})+\pi \xi\left(\phi_{j}\right)\right)
\end{aligned}
$$




$$
\begin{aligned}
& -\int_{\mathbb{R}^{d}} U\left((1-\pi) \xi(\tilde{f})+\pi \xi\left(\phi_{j}\right)-\xi\left(f^{*}\right)\right) \\
& \left.-\int_{\mathbb{R}^{d}} U\left(\xi\left(f^{*}\right)\right)\right]-\widehat{L}_{U}\left(f^{*}\right) \\
& =\sum_{j=1}^{N} p_{j} \widehat{L}_{U}\left(u\left((1-\pi) \xi(\tilde{f})+\pi \xi\left(\phi_{j}\right)-\xi\left(f^{*}\right)\right)\right) \\
& +\sum_{j=1}^{N} p_{j}\left[\int_{\mathbb{R}^{d}} U\left((1-\pi) \xi(\tilde{f})+\pi \xi\left(\phi_{j}\right)\right)\right. \\
& \left.-\int_{\mathbb{R}^{d}} U\left((1-\pi) \xi(\tilde{f})+\pi \xi\left(\phi_{j}\right)-\xi\left(f^{*}\right)\right)\right] \\
& -\int_{\mathbb{R}^{d}} U\left(\xi\left(f^{*}\right)\right) \\
& \equiv J_{1}+J_{2}+J_{3},
\end{aligned}
$$

where

$$
\begin{aligned}
& J_{1}=\sum_{j=1}^{N} p_{j} \widehat{L}_{U}\left(u\left((1-\pi)\left(\xi(\tilde{f})-\xi\left(f^{*}\right)\right)\right)\right), \\
& J_{2}=\sum_{j=1}^{N} p_{j} \widehat{L}_{U}\left(u\left(\pi\left(\xi\left(\phi_{j}\right)-\xi\left(f^{*}\right)\right)\right)\right)
\end{aligned}
$$

and

$$
\begin{aligned}
J_{3}= & \sum_{j=1}^{N} p_{j} \int_{\mathbb{R}^{d}} U\left((1-\pi)\left(\xi(\tilde{f})-\xi\left(f^{*}\right)\right)+\pi\left(\xi\left(\phi_{j}\right)-\xi\left(f^{*}\right)\right)\right) \\
& -\int_{\mathbb{R}^{d}} U\left((1-\pi)\left(\xi(\tilde{f})-\xi\left(f^{*}\right)\right)\right)-\sum_{j=1}^{N} p_{j} \int_{\mathbb{R}^{d}} U\left(\pi\left(\xi\left(\phi_{j}\right)-\xi\left(f^{*}\right)\right)\right) \\
& +\sum_{j=1}^{N} p_{j} \int_{\mathbb{R}^{d}}\left[U\left((1-\pi) \xi(\tilde{f})+\pi \xi\left(\phi_{j}\right)\right)\right. \\
& \left.-U\left((1-\pi) \xi(\tilde{f})+\pi \xi\left(\phi_{j}\right)-\xi\left(f^{*}\right)\right)\right] \\
& -\int_{\mathbb{R}^{d}} U\left(\xi\left(f^{*}\right)\right) .
\end{aligned}
$$

Now direct calculation gives

$$
\begin{aligned}
J_{1}= & -\frac{1}{n} \sum_{i=1}^{n}(1-\pi)\left[\xi\left(\tilde{f}\left(X_{i}\right)\right)-\xi\left(f^{*}\left(X_{i}\right)\right)\right] \\
& +\int_{\mathbb{R}^{d}} U\left((1-\pi)\left(\xi(\tilde{f})-\xi\left(f^{*}\right)\right)\right)
\end{aligned}
$$




$$
\begin{aligned}
= & -\frac{1}{n} \sum_{i=1}^{n}(1-\pi) \xi\left(\tilde{f}\left(X_{i}\right)\right)+\frac{1}{n} \sum_{i=1}^{n}(1-\pi) \xi\left(f^{*}\left(X_{i}\right)\right) \\
& +(1-\pi) \int_{\mathbb{R}^{d}} U(\xi(\tilde{f}))-(1-\pi) \int_{\mathbb{R}^{d}} U\left(\xi\left(f^{*}\right)\right) \\
& +\int_{\mathbb{R}^{d}} U\left((1-\pi)\left(\xi(\tilde{f})-\xi\left(f^{*}\right)\right)\right)-(1-\pi) \int_{\mathbb{R}^{d}} U(\xi(\tilde{f})) \\
& +(1-\pi) \int_{\mathbb{R}^{d}} U\left(\xi\left(f^{*}\right)\right) \\
= & (1-\pi)\left[\widehat{L}_{U}(\tilde{f})-\widehat{L}_{U}\left(f^{*}\right)\right]+\int_{\mathbb{R}^{d}} U\left((1-\pi)\left(\xi(\tilde{f})-\xi\left(f^{*}\right)\right)\right) \\
& -(1-\pi) \int_{\mathbb{R}^{d}} U(\xi(\tilde{f}))+(1-\pi) \int_{\mathbb{R}^{d}} U\left(\xi\left(f^{*}\right)\right) .
\end{aligned}
$$

Further we have

$$
\begin{aligned}
J_{2}= & \sum_{j=1}^{N} p_{j}\left[-\frac{1}{n} \sum_{i=1}^{n} \pi\left(\xi\left(\phi_{j}\left(X_{i}\right)\right)-\xi\left(f^{*}\left(X_{i}\right)\right)\right)\right. \\
& \left.+\int_{\mathbb{R}^{d}} U\left(\pi\left(\xi\left(\phi_{j}\right)-\xi\left(f^{*}\right)\right)\right)\right] \\
= & \sum_{j=1}^{N} p_{j} \int_{\mathbb{R}^{d}} U\left(\pi\left(\xi\left(\phi_{j}\right)-\xi\left(f^{*}\right)\right)\right)
\end{aligned}
$$

by the definition of $f^{*}$. Combining (11), (12), (13) and (14) furnishes to give

$$
\begin{aligned}
\sum_{j=1}^{N} p_{j} \widehat{L}_{U}\left(u\left((1-\pi) \xi(\tilde{f})+\pi \xi\left(\phi_{j}\right)\right)\right)-\widehat{L}_{U}\left(f^{*}\right) \\
=(1-\pi)\left[\widehat{L}_{U}(\tilde{f})-\widehat{L}_{U}\left(f^{*}\right)\right] \\
\quad+\sum_{j=1}^{N} p_{j} \int_{\mathbb{R}^{d}} U\left((1-\pi) \xi(\tilde{f})+\pi \xi\left(\phi_{j}\right)\right) \\
\quad-(1-\pi) \int_{\mathbb{R}^{d}} U(\xi(\tilde{f}))-\pi \int_{\mathbb{R}^{d}} U\left(\xi\left(f^{*}\right)\right) \\
=(1-\pi)\left[\widehat{L}_{U}(\tilde{f})-\widehat{L}_{U}\left(f^{*}\right)\right]+\theta\left(\pi \mid \tilde{f}, f^{*}\right) .
\end{aligned}
$$

The proof is complete.

Proof of Lemma 2 It is easily verified by the definitions of $\theta(\pi)$ and $\eta(\pi)$ in (8) and (9), and using the convex property of $U$.

Proof of Lemma 3 The convexity of $U$ gives

$$
U\left((1-\pi) \xi(\tilde{f}(x))+\pi \xi\left(\phi_{j}(x)\right)\right)-U\left((1-\pi) \xi(\tilde{f}(x))+\pi \xi\left(f^{*}(x)\right)\right)
$$




$$
\leq \pi u\left((1-\pi) \xi(\tilde{f}(x))+\pi \xi\left(\phi_{j}(x)\right)\right)\left(\xi\left(\phi_{j}(x)\right)-\xi\left(f^{*}(x)\right)\right) .
$$

Using this and the mean value theorem, we have

$$
\begin{aligned}
\eta(\pi) \leq & \pi \sum_{j=1}^{N} p_{j} \int_{\mathbb{R}^{d}} u\left((1-\pi) \xi(\tilde{f}(x))+\pi \xi\left(\phi_{j}(x)\right)\right)\left(\xi\left(\phi_{j}(x)\right)-\xi\left(f^{*}(x)\right)\right) d x \\
= & \pi \sum_{j=1}^{N} p_{j} \int_{\mathbb{R}^{d}}\left\{u(\xi(\tilde{f}(x)))+\pi\left(\xi\left(\phi_{j}(x)\right)-\xi(\tilde{f}(x))\right)\right. \\
& \left.\times U^{\prime \prime}\left(\xi(\tilde{f}(x))+\tau_{j} \pi\left(\xi\left(\phi_{j}(x)\right)-\xi(\tilde{f}(x))\right)\right)\right\}\left(\xi\left(\phi_{j}(x)\right)-\xi\left(f^{*}(x)\right)\right) d x \\
= & \pi^{2} \sum_{j=1}^{N} p_{j} \int_{\mathbb{R}^{d}} U^{\prime \prime}\left(\xi(\tilde{f}(x))+\tau_{j} \pi\left(\xi\left(\phi_{j}(x)\right)-\xi(\tilde{f}(x))\right)\right) \\
& \times\left(\xi\left(\phi_{j}(x)\right)-\xi(\tilde{f}(x))\right)\left(\xi\left(\phi_{j}(x)\right)-\xi\left(f^{*}(x)\right)\right) d x,
\end{aligned}
$$

for some $0<\tau_{j}<1(j=1, \ldots, N)$, where the second equality holds from the definition of $f^{*}$. Now there exists $1 \leq j_{0} \leq N$ such that

$$
\begin{aligned}
& \sum_{j=1}^{N} p_{j} \int_{\mathbb{R}^{d}} U^{\prime \prime}\left(\xi(\tilde{f}(x))+\tau_{j} \pi\left(\xi\left(\phi_{j}(x)\right)-\xi(\tilde{f}(x))\right)\right) \\
& \quad \times\left(\xi\left(\phi_{j}(x)\right)-\xi(\tilde{f}(x))\right)\left(\xi\left(\phi_{j}(x)\right)-\xi\left(f^{*}(x)\right)\right) d x \\
& \leq \int_{\mathbb{R}^{d}} U^{\prime \prime}\left(\xi(\tilde{f}(x))+\tau_{j_{0}} \pi\left(\xi\left(\phi_{j_{0}}(x)\right)-\xi(\tilde{f}(x))\right)\right) \\
& \quad \times\left(\xi\left(\phi_{j_{0}}(x)\right)-\xi(\tilde{f}(x))\right)\left(\xi\left(\phi_{j_{0}}(x)\right)-\xi\left(f^{*}(x)\right)\right) d x .
\end{aligned}
$$

Furthermore we see from the definitions of $\tilde{f}$ and $f^{*}$ that

$$
\begin{aligned}
\int_{\mathbb{R}^{d}} & U^{\prime \prime}\left(\xi(\tilde{f}(x))+\tau_{j_{0}} \pi\left(\xi\left(\phi_{j_{0}}(x)\right)-\xi(\tilde{f}(x))\right)\right) \\
& \times\left(\xi\left(\phi_{j_{0}}(x)\right)-\xi(\tilde{f}(x))\right)\left(\xi\left(\phi_{j_{0}}(x)\right)-\xi\left(f^{*}(x)\right)\right) d x \\
= & \sum_{\ell=1}^{k} \sum_{t=1}^{N} q_{\ell} p_{t} \int_{\mathbb{R}^{d}} U^{\prime \prime}\left(\left(1-\tau_{j_{0}} \pi\right) \sum_{l=1}^{k} q_{l} \xi\left(\tilde{\phi}_{l}(x)\right)+\tau_{j_{0}} \pi \xi\left(\phi_{j_{0}}(x)\right)\right) \\
& \times\left(\xi\left(\phi_{j_{0}}(x)\right)-\xi\left(\tilde{\phi}_{\ell}(x)\right)\right)\left(\xi\left(\phi_{j_{0}}(x)\right)-\xi\left(\phi_{t}(x)\right)\right) d x
\end{aligned}
$$

Put

$$
\tilde{\Phi}_{\ell}=\left(\sum_{l=1}^{k} q_{l} \xi\left(\tilde{\phi}_{l}\right), \phi_{j_{0}}, \tilde{\phi}_{\ell}\right) \in H(\mathcal{D}), \quad \ell=1, \ldots, k
$$

and

$$
\Phi_{t}=\left(\sum_{l=1}^{k} q_{l} \xi\left(\tilde{\phi}_{l}\right), \phi_{j_{0}}, \phi_{t}\right) \in H(\mathcal{D}), \quad t=1, \ldots, N
$$


Then noting the positiveness of $U^{\prime \prime}$, applying the Cauchy-Schwarz inequality and (5) yield

$$
\begin{aligned}
\sum_{\ell=1}^{k} & \sum_{t=1}^{N} q_{\ell} p_{t} \int_{\mathbb{R}^{d}} U^{\prime \prime}\left(\left(1-\tau_{j_{0}} \pi\right) \sum_{l=1}^{k} q_{l} \xi\left(\tilde{\phi}_{l}(x)\right)+\tau_{j_{0}} \pi\left(\xi\left(\phi_{j_{0}}(x)\right)\right)\right) \\
& \times\left(\xi\left(\phi_{j_{0}}(x)\right)-\xi\left(\tilde{\phi}_{\ell}(x)\right)\right)\left(\xi\left(\phi_{j_{0}}(x)\right)-\xi\left(\phi_{t}(x)\right)\right) d x \\
\leq & \sum_{\ell=1}^{k} \sum_{t=1}^{N} q_{\ell} p_{t} \int_{\mathbb{R}^{d}} U^{\prime \prime}\left(\left(1-\tau_{j_{0}} \pi\right) \sum_{l=1}^{k} q_{l} \xi\left(\tilde{\phi}_{l}(x)\right)+\tau_{j_{0}} \pi\left(\xi\left(\phi_{j_{0}}(x)\right)\right)\right) \\
& \times\left|\xi\left(\phi_{j_{0}}(x)\right)-\xi\left(\tilde{\phi}_{\ell}(x)\right)\right|\left|\xi\left(\phi_{j_{0}}(x)\right)-\xi\left(\phi_{t}(x)\right)\right| d x \\
\leq & \sum_{\ell=1}^{k} \sum_{t=1}^{N} q_{\ell} p_{t} \sqrt{\psi_{U}\left(\tau_{j_{0}} \pi \mid \tilde{\Phi}_{\ell}\right)} \sqrt{\psi_{U}\left(\tau_{j_{0}} \pi \mid \Phi_{t}\right)} \\
\leq & \sum_{\ell=1}^{k} \sum_{t=1}^{N} q_{\ell} p_{t} \sqrt{B_{U}^{2}} \sqrt{B_{U}^{2}} \\
= & B_{U}^{2} .
\end{aligned}
$$

This completes the proof.

Proof of Lemma 4 Let $0<\delta<B_{U}^{2}$ and let $f^{*} \in\{f(\cdot, \Lambda) \mid \Lambda \in \mathcal{W}\}$ be such that

$$
\widehat{L}_{U}\left(f^{*}\right) \leq \inf _{\Lambda \in \mathcal{W}} \widehat{L}_{U}(f(\cdot, \Lambda))+\delta .
$$

We are going to prove for $k=0,1, \ldots, M-1$ that

$$
\widehat{L}_{U}\left(\tilde{f}_{k}\right) \leq \widehat{L}_{U}\left(f^{*}\right)+\frac{\theta^{2} B_{U}^{2}}{k+\theta}+\varepsilon
$$

Note that, by (6), $f^{*}$ in (15) can be written as

$$
f^{*}=u\left(\sum_{i=1}^{N} p_{i} \xi\left(\phi_{i}\right)\right),
$$

for some $p_{i} \geq 0, \sum_{i=1}^{N} p_{i}=1$, and $\phi_{i} \in \mathcal{D}$. We have from Lemmas 1,2 , and 3 that

$$
\begin{aligned}
& \sum_{j=1}^{N} p_{j} \widehat{L}_{U}\left(u\left(\left(1-\pi_{k}\right) \xi\left(\tilde{f}_{k-1}\right)+\pi_{k} \xi\left(\phi_{j}\right)\right)\right)-\widehat{L}_{U}\left(f^{*}\right) \\
& \quad=\left(1-\pi_{k}\right)\left[\widehat{L}_{U}\left(\tilde{f}_{k-1}\right)-\widehat{L}_{U}\left(f^{*}\right)\right]+\theta\left(\pi_{k} \mid \tilde{f}_{k-1}, f^{*}\right) \\
& \quad \leq\left(1-\pi_{k}\right)\left[\widehat{L}_{U}\left(\tilde{f}_{k-1}\right)-\widehat{L}_{U}\left(f^{*}\right)\right]+\eta\left(\pi_{k} \mid \tilde{f}_{k-1}, f^{*}\right) \\
& \quad \leq\left(1-\pi_{k}\right)\left[\widehat{L}_{U}\left(\tilde{f}_{k-1}\right)-\widehat{L}_{U}\left(f^{*}\right)\right]+\pi_{k}^{2} B_{U}^{2} .
\end{aligned}
$$

Hence from (17), there exists $\phi^{*} \in\left\{\phi_{1}, \ldots, \phi_{N}\right\}$ such that

$$
\begin{gathered}
\widehat{L}_{U}\left(u\left(\left(1-\pi_{k}\right) \xi\left(\tilde{f}_{k-1}\right)+\pi_{k} \xi\left(\phi^{*}\right)\right)\right)-\widehat{L}_{U}\left(f^{*}\right) \\
\leq\left(1-\pi_{k}\right)\left[\widehat{L}_{U}\left(\tilde{f}_{k-1}\right)-\widehat{L}_{U}\left(f^{*}\right)\right]+\pi_{k}^{2} B_{U}^{2} .
\end{gathered}
$$


The Algorithm in Sect. 2 implies

$$
\widehat{L}_{U}\left(\tilde{f}_{k}\right)-\widehat{L}_{U}\left(f^{*}\right) \leq \widehat{L}_{U}\left(u\left(\left(1-\pi_{k}\right) \xi\left(\tilde{f}_{k-1}\right)+\pi_{k} \xi\left(\phi^{*}\right)\right)\right)-\widehat{L}_{U}\left(f^{*}\right)+\pi_{k} \varepsilon .
$$

Therefore we have from (18) and (19) that

$$
\widehat{L}_{U}\left(\tilde{f}_{k}\right)-\widehat{L}_{U}\left(f^{*}\right) \leq\left(1-\pi_{k}\right)\left[\widehat{L}_{U}\left(\tilde{f}_{k-1}\right)-\widehat{L}_{U}\left(f^{*}\right)\right]+\pi_{k}^{2} B_{U}^{2}+\pi_{k} \varepsilon .
$$

We are now in the position to prove (16) by induction. Referring to the definition of $\tilde{f}_{0}$ in the Algorithm, (18), $\pi_{0}=1$ and $\theta \geq 2$, it follows that

$$
\begin{aligned}
\widehat{L}_{U}\left(\tilde{f}_{0}\right) & \leq \widehat{L}_{U}\left(\phi^{*}\right)+\varepsilon \\
& \leq \widehat{L}_{U}\left(f^{*}\right)+B_{U}^{2}+\varepsilon \\
& \leq \widehat{L}_{U}\left(f^{*}\right)+\theta B_{U}^{2}+\varepsilon \\
& =\widehat{L}_{U}\left(f^{*}\right)+\frac{\theta^{2} B_{U}^{2}}{0+\theta}+\varepsilon
\end{aligned}
$$

this proves the case $k=0$. Next we assume for $k=t-1$ that

$$
\widehat{L}_{U}\left(\tilde{f}_{t-1}\right) \leq \widehat{L}_{U}\left(f^{*}\right)+\frac{\theta^{2} B_{U}^{2}}{(t-1)+\theta}+\varepsilon .
$$

Then using (20) and (21), we have

$$
\begin{aligned}
\widehat{L}_{U} & \left(\tilde{f}_{t}\right)-\widehat{L}_{U}\left(f^{*}\right) \\
& \leq\left(1-\pi_{t}\right)\left[\widehat{L}_{U}\left(\tilde{f}_{t-1}\right)-\widehat{L}_{U}\left(f^{*}\right)\right]+\pi_{t}^{2} B_{U}^{2}+\pi_{t} \varepsilon \\
& \leq\left(1-\pi_{t}\right)\left[\frac{\theta^{2} B_{U}^{2}}{(t-1)+\theta}+\varepsilon\right]+\frac{\theta^{2} B_{U}^{2}}{\{t+\theta\}^{2}}+\pi_{t} \varepsilon \\
& =\left[\frac{t}{t+\theta} \cdot \frac{\theta^{2}}{(t-1)+\theta}+\frac{\theta^{2}}{\{t+\theta\}^{2}}\right] B_{U}^{2}+\left\{\left(1-\pi_{t}\right)+\pi_{t}\right\} \varepsilon \\
& =\theta^{2}\left[\frac{t}{(t+\theta)\{(t-1)+\theta\}}+\frac{1}{(t+\theta)^{2}}\right] B_{U}^{2}+\varepsilon \\
& =\theta^{2}\left[\frac{(t+\theta)(t+1)-1}{(t+\theta)^{2}\{(t-1)+\theta\}}\right] B_{U}^{2}+\varepsilon \\
& \leq \theta^{2}\left[\frac{(t+\theta)(t+1)}{(t+\theta)^{2}\{(t-1)+\theta\}}\right] B_{U}^{2}+\varepsilon \\
& =\theta^{2} \frac{t+1}{(t+\theta)\{(t-1)+\theta\}} B_{U}^{2}+\varepsilon \\
& =\theta^{2} \frac{t+1}{(t+\theta)\{(t+1)+(\theta-2)\}} B_{U}^{2}+\varepsilon \\
& \leq \frac{\theta^{2}}{t+\theta} B_{U}^{2}+\varepsilon .
\end{aligned}
$$


Therefore the proof of (16) is complete. By putting $k=M-1$ and $\delta \rightarrow 0$, the assertion of Lemma holds.

Proof of Lemma 5 Let $\delta^{\prime}>0$ and let $f^{0} \in\{f(\cdot, \Lambda) \mid \Lambda \in \mathcal{W}\}$ be such that

$$
D_{U}\left(f, f^{0}\right) \leq \inf _{\Lambda \in \mathcal{W}} D_{U}(f, f(\cdot, \Lambda))+\delta^{\prime}
$$

Since

$$
\begin{aligned}
& D_{U}(f, g)-\widehat{L}_{U}(g) \\
& \quad=\frac{1}{n} \sum_{i=1}^{n} \xi\left(g\left(X_{i}\right)\right)-\int_{\mathbb{R}^{d}} f(x) \xi(g(x)) d x-\int_{\mathbb{R}^{d}}\{U(\xi(f(x)))-f(x) \xi(f(x))\} d x,
\end{aligned}
$$

we have

$$
\begin{aligned}
D_{U}(f, \hat{f})-\widehat{L}_{U}(\hat{f})= & \frac{1}{n} \sum_{i=1}^{n} \xi\left(\hat{f}\left(X_{i}\right)\right)-\int_{\mathbb{R}^{d}} f(x) \xi(\hat{f}(x)) d x \\
& -\int_{\mathbb{R}^{d}}\{U(\xi(f(x)))-f(x) \xi(f(x))\} d x, \\
D_{U}\left(f, f^{0}\right)-\widehat{L}_{U}\left(f^{0}\right)= & \frac{1}{n} \sum_{i=1}^{n} \xi\left(f^{0}\left(X_{i}\right)\right)-\int_{\mathbb{R}^{d}} f(x) \xi\left(f^{0}(x)\right) d x \\
& -\int_{\mathbb{R}^{d}}\{U(\xi(f(x)))-f(x) \xi(f(x))\} d x .
\end{aligned}
$$

Subtracting both side gives

$$
\begin{aligned}
& D_{U}(f, \hat{f})-\widehat{L}_{U}(\hat{f})-D_{U}\left(f, f^{0}\right)+\widehat{L}_{U}\left(f^{0}\right) \\
& \quad=\frac{1}{n} \sum_{i=1}^{n} \xi\left(\hat{f}\left(X_{i}\right)\right)-\frac{1}{n} \sum_{i=1}^{n} \xi\left(f^{0}\left(X_{i}\right)\right)-\int_{\mathbb{R}^{d}} f(x) \xi(\hat{f}(x)) d x+\int_{\mathbb{R}^{d}} f(x) \xi\left(f^{0}(x)\right) d x \\
& \quad=v_{n}\left(\xi(\hat{f})-\xi\left(f^{0}\right)\right) .
\end{aligned}
$$

Hence, using assumption, we have

$$
\begin{aligned}
& D_{U}(f, \hat{f})-D_{U}\left(f, f^{0}\right) \\
& \quad=D_{U}(f, \hat{f})-\widehat{L}_{U}(\hat{f})+\widehat{L}_{U}(\hat{f})-D_{U}\left(f, f^{0}\right) \\
& \quad \leq D_{U}(f, \hat{f})-\widehat{L}_{U}(\hat{f})+\widehat{L}_{U}\left(f^{0}\right)-D_{U}\left(f, f^{0}\right)+\tau \\
& \quad=v_{n}\left(\xi(\hat{f})-\xi\left(f^{0}\right)\right)+\tau .
\end{aligned}
$$

Now direct calculations yield that

$$
\int_{\mathbb{R}^{d}} f(x) \xi(\hat{f}(x)) d x=\int_{\mathbb{R}^{d}} f(x) \xi\left(u\left(\sum_{\phi \in \mathcal{D}} \hat{\lambda}_{\phi} \xi(\phi(x))\right)\right) d x
$$




$$
\begin{aligned}
& =\int_{\mathbb{R}^{d}} f(x)\left\{\sum_{\phi \in \mathcal{D}} \hat{\lambda}_{\phi} \xi(\phi(x))\right\} d x \\
& =\sum_{\phi \in \mathcal{D}} \hat{\lambda}_{\phi} \int_{\mathbb{R}^{d}} f(x) \xi(\phi(x)) d x,
\end{aligned}
$$

and

$$
\begin{aligned}
\frac{1}{n} \sum_{i=1}^{n} \xi\left(\hat{f}\left(X_{i}\right)\right) & =\frac{1}{n} \sum_{i=1}^{n} \xi\left(u\left(\sum_{\phi \in \mathcal{D}} \hat{\lambda}_{\phi} \xi\left(\phi\left(X_{i}\right)\right)\right)\right) \\
& =\frac{1}{n} \sum_{i=1}^{n} \sum_{\phi \in \mathcal{D}} \hat{\lambda}_{\phi} \xi\left(\phi\left(X_{i}\right)\right) \\
& =\sum_{\phi \in \mathcal{D}} \hat{\lambda}_{\phi}\left\{\frac{1}{n} \sum_{i=1}^{n} \xi\left(\phi\left(X_{i}\right)\right)\right\},
\end{aligned}
$$

where $\hat{f}(x)=f\left(x, \hat{\Lambda}_{n}\right)$ for $\hat{\Lambda}_{n}=\left(\hat{\lambda}_{\phi}\right)_{\phi \in \mathcal{D}}$. Similarly we have

$$
\int_{\mathbb{R}^{d}} f(x) \xi\left(f^{0}(x)\right) d x=\sum_{\phi \in \mathcal{D}} \lambda_{\phi}^{0} \int_{\mathbb{R}^{d}} f(x) \xi(\phi(x)) d x
$$

and

$$
\frac{1}{n} \sum_{i=1}^{n} \xi\left(f^{0}\left(X_{i}\right)\right)=\sum_{\phi \in \mathcal{D}} \lambda_{\phi}^{0}\left\{\frac{1}{n} \sum_{i=1}^{n} \xi\left(\phi\left(X_{i}\right)\right)\right\}
$$

where $f^{0}(x)=f\left(x, \Lambda^{0}\right)$ for $\Lambda^{0}=\left(\lambda_{\phi}^{0}\right)_{\phi \in \mathcal{D}}$. Let us define the vectors of the empirical and theoretical coefficients as

$$
\hat{\Theta}_{n}=\left(\frac{1}{n} \sum_{i=1}^{n} \xi\left(\phi\left(X_{i}\right)\right)\right)_{\phi \in \mathcal{D}} \quad \text { and } \quad \Theta_{f}=\left(\int_{\mathbb{R}^{d}} f(x) \xi(\phi(x)) d x\right)_{\phi \in \mathcal{D}} .
$$

Then

$$
\begin{aligned}
v_{n} & \left(\xi(\hat{f})-\xi\left(f^{0}\right)\right) \\
& =\left(\hat{\Theta}_{n}-\Theta_{f}\right)^{T} \hat{\Lambda}_{n}-\left(\hat{\Theta}_{n}-\Theta_{f}\right)^{T} \Lambda^{0} \\
& =\left(\hat{\Lambda}_{n}-\Lambda^{0}\right)^{T}\left(\hat{\Theta}_{n}-\Theta_{f}\right) \\
& \leq\left\{\left\|\hat{\Lambda}_{n}\right\|_{\ell_{1}}+\left\|\Lambda^{0}\right\|_{\ell_{1}}\right\}\left\|\hat{\Theta}_{n}-\Theta_{f}\right\|_{\ell_{\infty}} \\
& \leq 2\left\|\hat{\Theta}_{n}-\Theta_{f}\right\|_{\ell_{\infty}} \\
& =2 \sup _{\phi \in \mathcal{D}}\left|v_{n}(\xi(\phi))\right| .
\end{aligned}
$$

Here we have used the notation

$$
\|\Lambda\|_{\ell_{1}}=\sum_{\phi \in \mathcal{D}}\left|\lambda_{\phi}\right| \quad \text { and } \quad\|\Lambda\|_{\ell_{\infty}}=\sup _{\phi \in \mathcal{D}}\left|\lambda_{\phi}\right|
$$


for $\Lambda=\left(\lambda_{\phi}\right)_{\phi \in \mathcal{D}}$. Therefore

$$
\begin{aligned}
D_{U}(f, \hat{f}) & \leq D_{U}\left(f, f^{0}\right)+v_{n}\left(\xi(\hat{f})-\xi\left(f^{0}\right)\right)+\tau \\
& \leq D_{U}\left(f, f^{0}\right)+2 \sup _{\phi \in \mathcal{D}}\left|v_{n}(\xi(\phi))\right|+\tau \\
& \leq \inf _{\Lambda \in \mathcal{W}} D_{U}(f, f(\cdot, \Lambda))+\delta^{\prime}+2 \sup _{\phi \in \mathcal{D}}\left|v_{n}(\xi(\phi))\right|+\tau .
\end{aligned}
$$

The assertion of lemma holds by $\delta^{\prime} \rightarrow 0$.

Proof of Lemma 6 We see from the definition of $D_{U}$ in (1) that

$$
\begin{aligned}
& D_{U}\left(f, g_{c}\right)-D_{U}(f, g) \\
& \quad=\int_{\mathbb{R}^{d}}\left[U\left(\xi\left(g_{c}(x)\right)\right)-U(\xi(g(x)))-f(x)\left\{\xi\left(g_{c}(x)\right)-\xi(g(x))\right\}\right] d x
\end{aligned}
$$

Since $U$ is convex, it holds that $U(a)-U(b) \geq U^{\prime}(b)(a-b)$ for any $a, b \in \mathbb{R}$, which implies $U(b)-U(a) \leq U^{\prime}(b)(b-a)$. By applying this property and $U^{\prime}=u=\xi^{-1}$, we have

$$
\begin{aligned}
\int_{\mathbb{R}^{d}} & {\left[U\left(\xi\left(g_{c}(x)\right)\right)-U(\xi(g(x)))\right] d x } \\
& -\int_{\mathbb{R}^{d}} f(x)\left\{\xi\left(g_{c}(x)\right)-\xi(g(x))\right\} d x \\
\leq & \int_{\mathbb{R}^{d}}\left[U^{\prime}\left(\xi\left(g_{c}(x)\right)\right)\left\{\xi\left(g_{c}(x)\right)-\xi(g(x))\right\}\right] d x \\
& -\int_{\mathbb{R}^{d}} f(x)\left\{\xi\left(g_{c}(x)\right)-\xi(g(x))\right\} d x \\
= & \int_{\mathbb{R}^{d}}\left\{g_{c}(x)-f(x)\right\}\left\{\xi\left(g_{c}(x)\right)-\xi(g(x))\right\} d x .
\end{aligned}
$$

Now the mean value theorem and the differential of the inverse function give

$$
\begin{aligned}
\xi\left(g_{c}(x)\right)-\xi(g(x)) & =\xi^{\prime}\left(g(x)+\theta_{x}\left(g_{c}(x)-g(x)\right)\right)\left\{g_{c}(x)-g(x)\right\} \\
& =\frac{1}{u^{\prime}\left(u^{-1}\left(g(x)+\theta_{x}\left(g_{c}(x)-g(x)\right)\right)\right)}\left(v_{g}^{-1}-1\right) g(x) \\
& =\left(v_{g}^{-1}-1\right) \frac{g(x)}{U^{\prime \prime}\left(\xi\left(g(x)+\theta_{x}\left(g_{c}(x)-g(x)\right)\right)\right)}
\end{aligned}
$$

for some $0<\theta_{x}<1$. Combining (22), (23), (24) and Assumption 2 yield that

$$
\begin{aligned}
& D_{U}\left(f, g_{c}\right)-D_{U}(f, g) \\
& \quad \leq\left(v_{g}^{-1}-1\right) \int_{\mathbb{R}^{d}}\left\{g_{c}(x)-f(x)\right\} \frac{g(x)}{U^{\prime \prime}\left(\xi\left(g(x)+\theta_{x}\left(g_{c}(x)-g(x)\right)\right)\right)} d x \\
& \quad \leq\left|v_{g}^{-1}-1\right| \int_{\mathbb{R}^{d}}\left|g_{c}(x)-f(x)\right| \frac{g(x)}{\left.U^{\prime \prime}\left(\xi\left(\theta_{x} g_{c}(x)+\left(1-\theta_{x}\right) g(x)\right)\right)\right)} d x
\end{aligned}
$$




$$
\begin{aligned}
& \leq\left|v_{g}^{-1}-1\right| \int_{\mathbb{R}^{d}}\left|g_{c}(x)-f(x)\right| \frac{g(x)}{\left.\inf _{\delta \in[0,1]} U^{\prime \prime}\left(\xi\left(\delta g_{c}(x)+(1-\delta) g(x)\right)\right)\right)} d x \\
& \leq\left|v_{g}^{-1}-1\right| \int_{\mathbb{R}^{d}}\left|g_{c}(x)-f(x)\right| \frac{g(x)}{C_{U} g(x)^{\alpha}} d x \\
& =C_{U}^{-1}\left|v_{g}^{-1}-1\right| \int_{\mathbb{R}^{d}}\left|g_{c}(x)-f(x)\right| g(x)^{1-\alpha} d x,
\end{aligned}
$$

which completes the proof.

\section{Appendix C: Proof of Theorems}

Proof of Theorem 3 Theorem 3 follows by the direct use of Lemma 5.

Proof of Theorem 1 Combining Lemma 4 and Lemma 5 with

$$
\tau=\frac{\theta^{2} B_{U}^{2}}{M+(\theta-1)}+\varepsilon
$$

yields Theorem 1.

Proof of Theorem 2 Theorem 2 follows using Lemma 6.

\section{References}

Basu, A., Harris, I. R., Hjort, N. L., \& Jones, M. C. (1998). Robust and efficient estimation by minimising a density power divergence. Biometrika, 85, 549-559.

Bishop, C. M. (2006). Pattern recognition and machine learning. Berlin: Springer.

Bühlman, P., \& Yu, B. (2003). Boosting with $L_{2}$ loss: regression and classification. Journal of the American Statistical Association, 98, 324-339.

Devroye, L., Györfi, L., \& Lugosi, G. (1996). A probabilistic theory of pattern recognition. Berlin: Springer.

Di Marzio, M., \& Taylor, C. C. (2004). Boosting kernel density estimates: a bias reduction technique? Biometrika, 91, 226-233.

Duong, T. (2010). Reference manual for the package ks. http://cran.r-project.org/.

Duong, T., \& Hazelton, M. L. (2003). Plug-in bandwidth matrices for bivariate kernel density estimation. Journal of Nonparametric Statistics, 15, 17-30.

Duong, T., \& Hazelton, M. L. (2005a). Convergence rates for unconstrained bandwidth matrix selectors in multivariate kernal density estimation. Journal of Multivariate Analysis, 93, 417-433.

Duong, T., \& Hazelton, M. L. (2005b). Cross-validation bandwidth matrices for multivariate kernel density estimation. Scandinavian Journal of Statistics, 38, 485-506.

Eguchi, S. (2008). Information divergence geometry and the application to statistical machine learning. In F. Emmert-Streib \& M. Dehmer (Eds.), Information theory and statistical learning (pp. 309-332). Berlin: Springer.

Friedman, J. H., Stuetzle, W., \& Schroeder, A. (1984). Projection pursuit density estimation. Journal of the American Statistical Association, 79, 599-608.

Fujisawa, H., \& Eguchi, S. (2008). Robust parameter estimation with a small bias against heavy contamination. Journal of Multivariate Analysis, 99, 2053-2081.

Girolami, M., \& He, C. (2003). Probability density estimation from optimally condensed data samples. IEEE Transactions on Pattern Analysis and Machine Intelligence, 25, 1253-1264.

Hastie, T., Tibishirani, R., \& Friedman, J. H. (2009). The elements of statistical learning: data mining, inference and prediction (2nd ed.). New York: Springer. 
Higuchi, I., \& Eguchi, S. (2004). Robust principal component analysis with adaptive selection for tuning parameters. Journal of Machine Learning Research, 5, 453-471.

Jones, M. C., Marron, J. S., \& Sheather, S. J. (1996). A brief survey of bandwidth selection for density estimation. Journal of the American Statistical Association, 91, 401-407.

Klemelä, J. (2007). Density estimation with stagewise optimization of the empirical risk. Machine Learning, 67, 169-195

Klemelä, J., \& Mammen, E. (2010). Empirical risk minimization in inverse problems. Annals of Statistics, $38,482-511$.

Minami, M., \& Eguchi, S. (2002). Robust blind source separation by beta-divergence. Neural Computation, 14, 1859-1886.

Murata, N., Takenouchi, T., Kanamori, T., \& Eguchi, S. (2004). Information geometry of U-boost and Bregman divergence. Neural Computation, 16, 1437-1481.

Ridgeway, G. (2002). Looking for lumps: boosting and bagging for density estimation. Computational Statistics \& Data Analysis, 38, 379-392.

Rockafeller, R. T. (1996). Convex analysis. Princeton: Princeton University Press.

Rosset, S., \& Segal, E. (2002). Boosting density estimation. In Proceedings of the 16th international conference on neural information processing systems (NIPS).

Schapire, R. E. (1990). The strength of weak learnability. Machine Learning, 5, 197-227.

Scott, D. W. (2001). Parametric statistical modeling by minimum integrated square error. Technometrics, 43, 274-285.

Simonoff, J. S. (1996). Smoothing methods in statistics. Berlin: Springer.

Silverman, B. W. (1986). Density estimation for statistics and data analysis. London: Chapman and Hall.

Wand, M. P., \& Jones, M. C. (1993). Comparison of smoothing parameterizations in bivariate kernel density estimation. Journal of the American Statistical Association, 88, 520-528. 